\title{
№ 6
}

Informe Especial
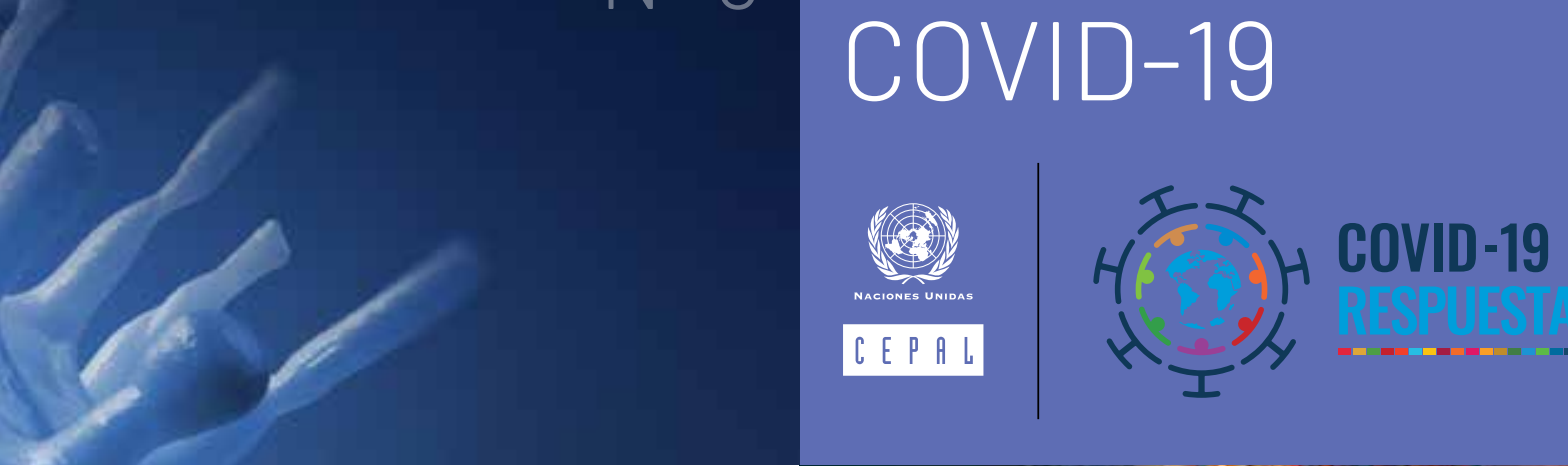

\section{Los efectos del COVID-19 en el comercio internacional y la logística}

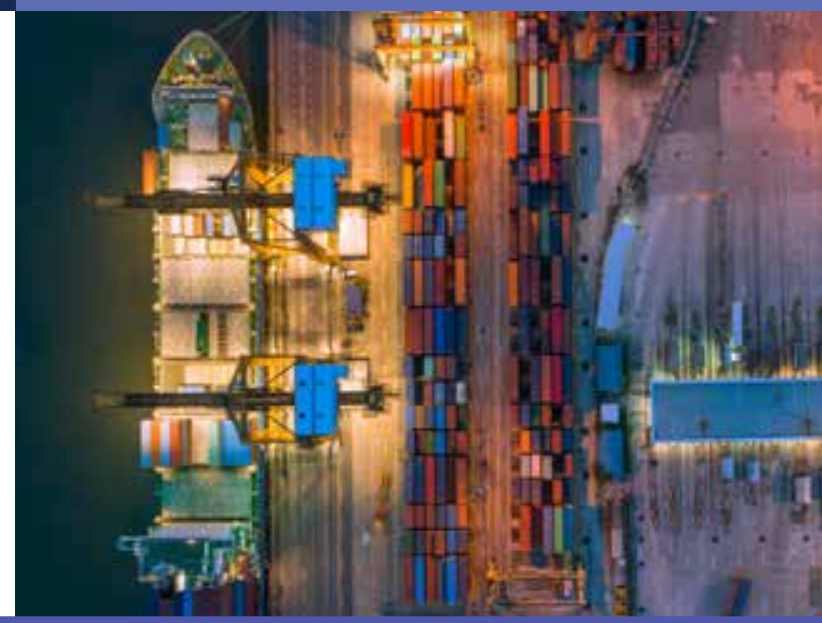

\section{A. Se desploma el comercio mundial}

- La irrupción del COVID-19 se produjo en un contexto de debilitamiento del comercio mundial que se arrastra desde la crisis financiera de 2008-2009. Mientras que entre 1990 y 2007 el volumen del comercio de bienes se expandió a una tasa media del 6,2\% anual, entre 2012 y 2019 lo hizo apenas a una del 2,3\% anual (véase el gráfico 1). Asimismo, la participación de las exportaciones de bienes y servicios en el PIB mundial, que alcanzó su máximo histórico del 31\% en 2008 , desde 2015 se ha ubicado en torno al $28 \%$.

Gráfico 1 | Tasa de variación del volumen del comercio mundial de bienes, 1990-2019 (En porcentajes)

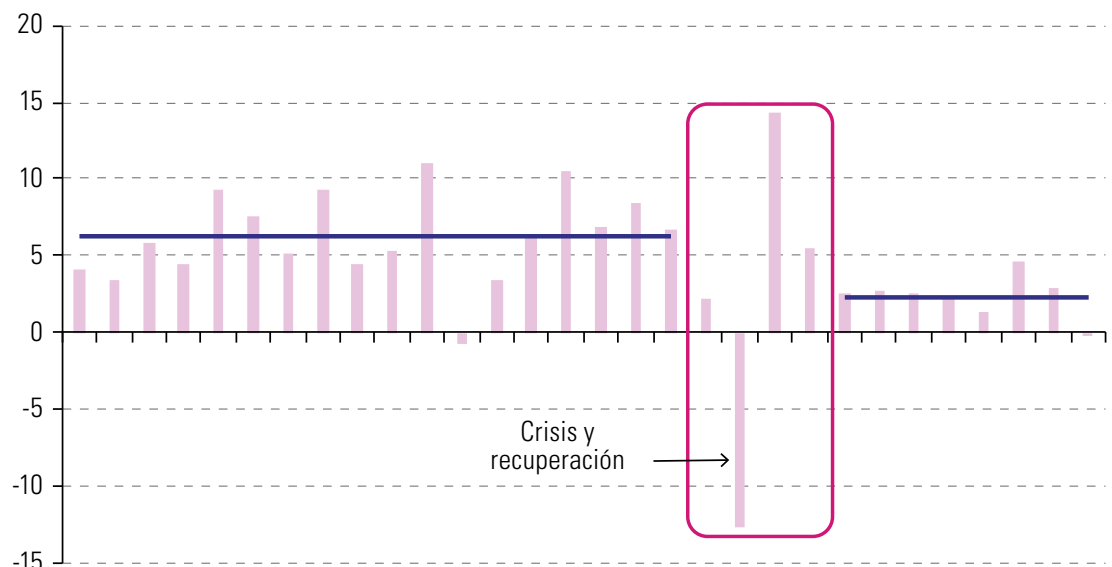

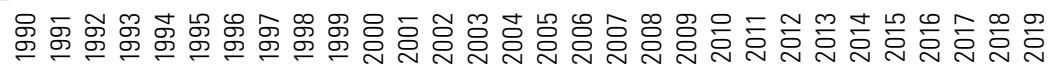

$$
\text { — Variación media Variación anual }
$$

Fuente: Comisión Económica para América Latina y el Caribe (CEPAL), sobre la base de información de la Organización Mundial del Comercio (OMC).

\section{Índice}

A. Se desploma el comercio mundial ...... 1

B. El comercio de bienes de la región cayó un $17 \%$ entre enero y mayo de 2020. 3

C. El colapso del turismo arrastra a las exportaciones de servicios.

D. Los problemas en el transporte internacional traban el comercio regional 14

E. Las exportaciones y las importaciones de bienes se reducirían en una cuarta parte en 2020

F. Ante una economía mundial más incierta y regionalizada, es imperativo profundizar la integración regional 22

Bibliografía 23 
La rápida propagación del COVID-19 y las medidas adoptadas por los gobiernos han tenido graves consecuencias en las principales economías mundiales. Se ha interrumpido gran parte de las actividades productivas, primero en Asia y posteriormente en Europa, América del Norte y el resto del mundo, y ha habido cierres generalizados de fronteras. Esto ha dado lugar a un marcado aumento del desempleo, especialmente en los Estados Unidos, con la consecuente reducción de la demanda de bienes y servicios. En este contexto, en 2020 el producto mundial registraría su mayor contracción desde la Segunda Guerra Mundial (Banco Mundial, 2020).

- En esta coyuntura, en mayo de 2020 el volumen del comercio mundial de bienes cayó un $17,7 \%$ con respecto al mismo mes de 2019 (véase el gráfico 2). La caída en los primeros cinco meses del año fue generalizada, si bien afectó especialmente a las exportaciones de los Estados Unidos, el Japón y la Unión Europea (véase el cuadro 1). China experimentó una contracción menor que el promedio mundial, ya que controló el brote y reabrió su economía relativamente rápido. América Latina y el Caribe es la región en desarrollo más afectada.

Gráfico 2 | Variación interanual del volumen del comercio mundial de bienes, enero de 2017 a mayo de 2020 (En porcentajes)

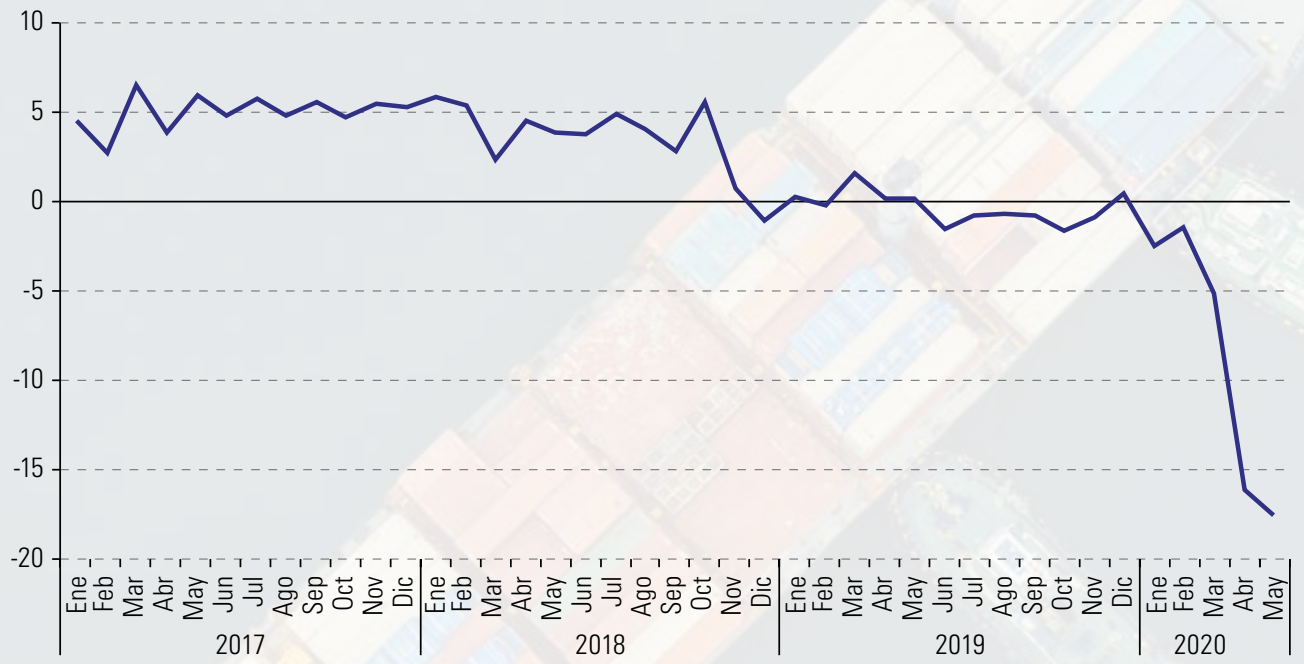

Fuente: Comisión Económica para América Latina y el Caribe (CEPAL), sobre la base Netherlands Bureau for Economic Policy Analysis (CPB), World Trade Monitor [base de datos en línea] https://www.cpb.nl/en/worldtrademonitor.

Cuadro 1 | Mundo, agrupaciones y países seleccionados: variación del volumen del comercio mundial de bienes, diciembre de 2019 a mayo de 2020

(En porcentajes)

\begin{tabular}{lcc}
\hline & Exportaciones & Importaciones \\
\hline Mundo & $-18,3$ & $-15,8$ \\
\hline Economías avanzadas & $-22,7$ & $-15,8$ \\
\hline Estados Unidos & $-30,8$ & $-15,8$ \\
\hline Japón & $-22,1$ & $-4,4$ \\
\hline Zona del euro & $-22,0$ & $-16,6$ \\
\hline Economías emergentes & $-12,8$ & $-15,8$ \\
\hline China & $-7,7$ & $-13,8$ \\
\hline Países emergentes de Asia (no incluye China) & $-13,1$ & $-17,2$ \\
\hline Europa Oriental y Comunidad de Estados Independientes (CEI) & $-4,4$ & $-13,4$ \\
\hline América Latina y el Caribe & $-26,1$ & $-27,4$ \\
\hline África y Oriente Medio & $-13,9$ & $-2,5$ \\
\hline
\end{tabular}

Fuente: Comisión Económica para América Latina y el Caribe (CEPAL), sobre la base Netherlands Bureau for Economic Policy Analysis (CPB), World Trade Monitor [base de datos en línea] https://www.cpb.nl/en/worldtrademonitor. 
Las cadenas de valor mundiales fueron el principal canal de transmisión de los efectos del COVID-19 al comercio mundial. Las medidas adoptadas por China en enero (cierre temporal de la provincia de Hubei y de las fronteras del país) implicaron la suspensión de exportaciones de insumos para industrias como la automotriz, electrónica, farmacéutica y de suministros médicos. Esto obligó a la paralización por varias semanas de fábricas en América del Norte, Europa y el resto de Asia que no disponían de proveedores alternativos, pues China es el principal exportador mundial de partes y componentes, con un $15 \%$ de los envíos mundiales en 2018.

- A partir de marzo, China reabrió gradualmente su economía y avanzó hacia la normalización de las exportaciones. Sin embargo, el choque inicial de oferta sobre el comercio mundial se intensificó gradualmente con un choque de demanda, producto de las medidas para minimizar el contagio adoptadas en Europa y posteriormente en América del Norte y el resto del mundo.

- La contracción del volumen del comercio mundial de bienes fue cercana al 18,5\% en el segundo trimestre de 2020 en comparación con igual período de 2019 (OMC, 2020a). Así, la caída durante el primer semestre se habría situado en alrededor del $11 \%$. Ello sugiere que la contracción del comercio mundial en $\mathbf{2 0 2 0}$ se ubicaría más cerca del extremo "optimista" del rango que va del $-13 \%$ (similar a la caída anual en 2009 ) al $-32 \%$ proyectado en abril. Estas proyecciones dependen claramente de la evolución de la pandemia durante el segundo semestre, particularmente en los Estados Unidos, donde las tasas de contagio siguen en aumento.

- El comercio de servicios también se ha visto gravemente afectado. El valor de las exportaciones de un grupo de 37 países, que en 2019 representaron alrededor de dos terceras partes de las exportaciones mundiales de servicios, se contrajo un $10,4 \%$ en el primer cuatrimestre de 2020 en comparación con el mismo período de 2019. El turismo, que en 2019 representó el $\mathbf{2 4 \%}$ de las exportaciones mundiales de servicios, ha sido particularmente afectado. Entre enero y abril de 2020 el número de turistas internacionales a nivel mundial disminuyó un 44\% en comparación con el mismo período de 2019. Para el año completo, las proyecciones indican una reducción de entre un 58\% y un $78 \%$, dependiendo de la evolución de la pandemia y del ritmo de relajamiento de las restricciones de viaje (OMT, 2020).

\section{B. El comercio de bienes de la región cayó un $17 \%$ entre enero y mayo de 2020}

El valor de las exportaciones e importaciones de bienes se redujo un $17 \%$ entre enero y mayo de 2020 en comparación con el mismo período de 2019. Ambos flujos se desplomaron hacia el final del período de cinco meses en 2020 , con una caída interanual del $37 \%$ en mayo. La disminución interanual del volumen del comercio en el bimestre abril-mayo de 2020 fue mucho mayor que en igual período de 2009, durante la crisis financiera: $20 \%$ las exportaciones y $25 \%$ las importaciones (véase el gráfico 3). En el caso de las exportaciones, ello refleja tanto un choque de oferta (el cierre parcial del aparato productivo) como de demanda (contracción económica en los principales mercados de la región). En el caso de las importaciones, su descenso obedece fundamentalmente a la profunda recesión por la que atraviesa la región, con una contracción esperada de su producto del 9,1\% (CEPAL, 2020a). 
Gráfico 3 América Latina y el Caribe: variación interanual de las exportaciones e importaciones de bienes según volumen, precio y valor, enero de 2007 a mayo de 2020

(En porcentajes)

\section{A. Exportaciones}

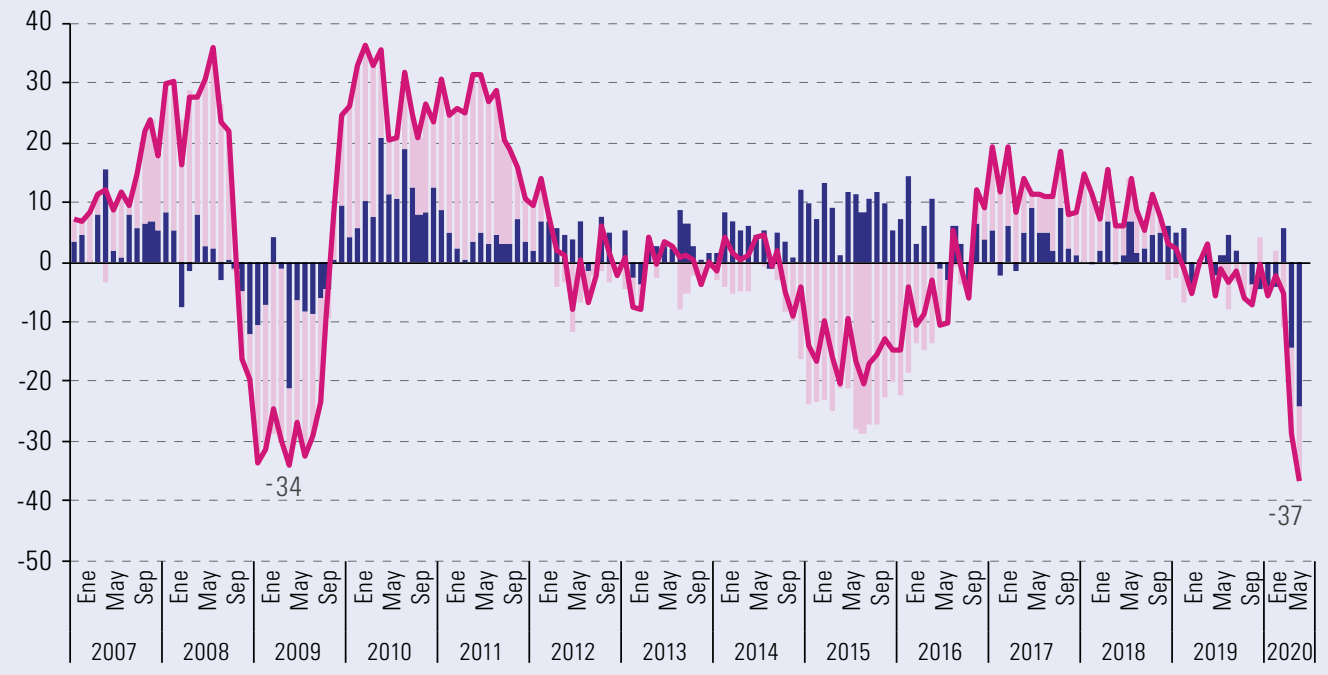

B. Importaciones

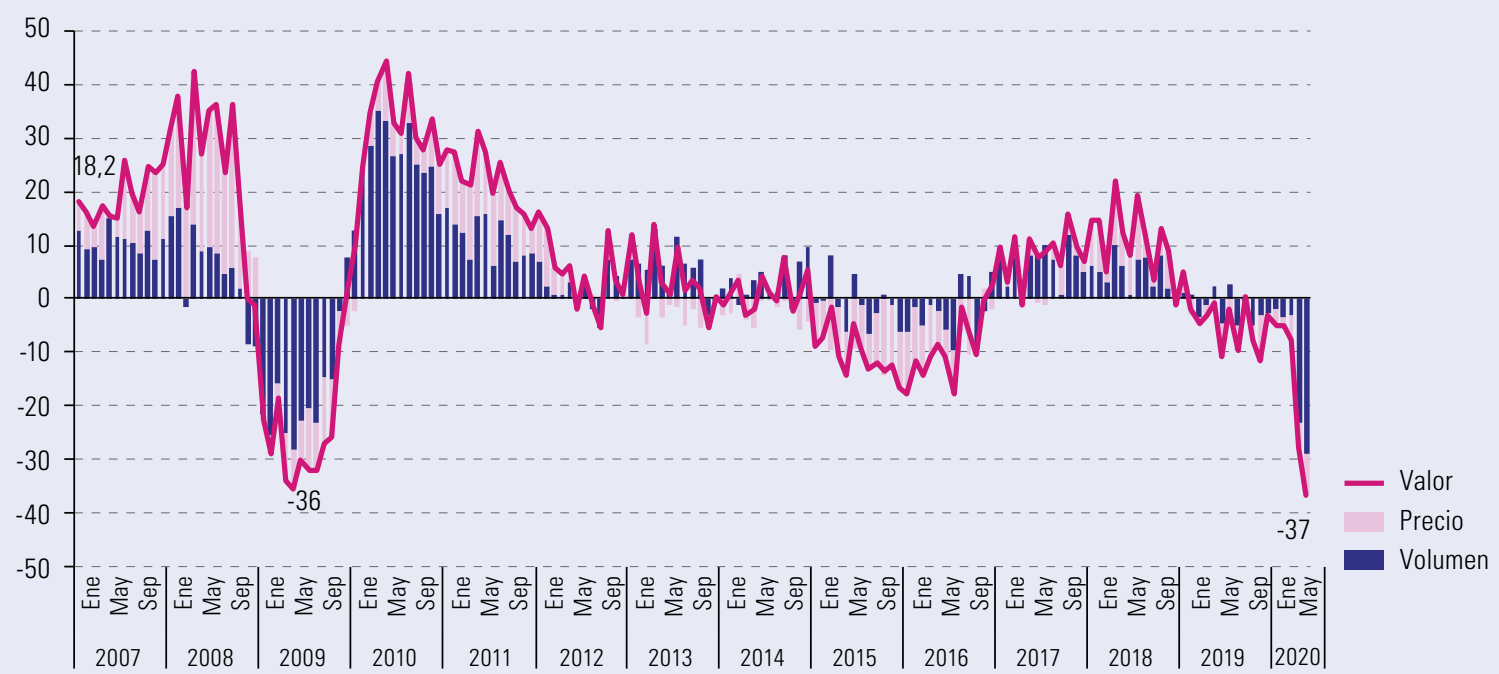

Fuente: Comisión Económica para América Latina y el Caribe (CEPAL), sobre la base de información de los bancos centrales, servicios de aduanas e institutos de estadística de los países.

\section{Dinámica de las exportaciones según países y bloques de integración}

- La caída del valor de las exportaciones regionales de bienes entre enero y mayo de 2020 se explica por una reducción del volumen del $\mathbf{8 , 6} \%$ y de los precios del $\mathbf{8} \%$, desplome que agudiza la pérdida de dinamismo de las exportaciones desde 2019 (véase el cuadro 2). En ese año, los envíos sufrieron los efectos de las tensiones comerciales entre los Estados Unidos y China y de los bajos precios de los minerales y metales. 
Cuadro 2 | América Latina y el Caribe: variación interanual del valor de las exportaciones de bienes, enero-mayo de 2018 a enero-mayo de 2020, y abril y mayo de 2020 en comparación con igual mes del año anterior (En porcentajes)

\begin{tabular}{|c|c|c|c|c|c|}
\hline Región/Subregión/Pais & $\begin{array}{c}\text { Enero-mayo } \\
2018\end{array}$ & $\begin{array}{c}\text { Enero-mayo } \\
2019\end{array}$ & $\begin{array}{c}\text { Enero-mayo } \\
2020\end{array}$ & Abril 2020 & Mayo 2020 \\
\hline América Latina y el Caribe & 10,7 & $-0,3$ & $-16,6$ & $-29,5$ & $-37,1$ \\
\hline Mercado Común del Sur (MERCOSUR) & 5,8 & $-4,1$ & $-12,4$ & $-11,7$ & $-16,2$ \\
\hline Argentina & 7,0 & 3,2 & $-11,5$ & $-18,4$ & $-15,9$ \\
\hline Brasil & 6,6 & $-2,1$ & $-6,5$ & $-5,8$ & $-13,1$ \\
\hline Paraguaya & 7,5 & $-18,4$ & $-8,2$ & $-18,8$ & $-11,2$ \\
\hline Uruguay & 4,5 & 1,0 & $-21,4$ & $-21,0$ & $-38,1$ \\
\hline Venezuela (República Bolivariana de) & $-1,0$ & $-27,0$ & $-65,0$ & $-53,1$ & $-47,7$ \\
\hline Comunidad Andina (CAN) & 16,6 & $-2,8$ & $-23,1$ & $-52,8$ & $-41,4$ \\
\hline Bolivia (Estado Plurinacional de) & 24,3 & $-8,7$ & $-23,8$ & $-62,1$ & $-58,5$ \\
\hline Colombia & 14,5 & 0,4 & $-25,0$ & $-52,3$ & $-40,6$ \\
\hline Ecuador & 13,6 & 3,1 & $-15,2$ & $-44,4$ & $-27,2$ \\
\hline Perú & 18,5 & $-7,3$ & $-25,3$ & $-56,3$ & $-36,3$ \\
\hline Alianza del Pacífico ${ }^{b}$ & 13,9 & 1,7 & $-19,8$ & $-37,6$ & $-49,4$ \\
\hline Chile & 21,3 & $-6,6$ & $-8,8$ & $-6,3$ & $-15,2$ \\
\hline México & 12,2 & 4,3 & $-20,8$ & $-40,7$ & $-56,7$ \\
\hline Mercado Común Centroamericano (MCCA) & 2,5 & $-1,8$ & 0,4 & $-13,9$ & $-8,0$ \\
\hline Costa Rica & 7,0 & 1,3 & 2,2 & $-11,6$ & $-3,6$ \\
\hline El Salvador & 5,2 & $-1,0$ & $-23,6$ & $-51,0$ & $-31,3$ \\
\hline Guatemala & $-1,3$ & $-1,9$ & 3,2 & $-8,1$ & $-1,6$ \\
\hline Honduras & $-1,2$ & $-8,5$ & 2,3 & $-3,5$ & $-5,7$ \\
\hline Nicaragua & 0,0 & $-4,1$ & 14,1 & 14,7 & 14,6 \\
\hline Panamác & 11,7 & 0,7 & $-11,5$ & $-30,8$ & $-52,3$ \\
\hline Países del Caribe & 11,2 & 4,5 & $-10,0$ & $-32,1$ & $-33,3$ \\
\hline Cuba & $-32,1$ & 9,5 & $-29,8$ & $-38,3$ & $-51,6$ \\
\hline República Dominicana & 7,8 & 4,9 & $-8,5$ & $-27,4$ & $-28,4$ \\
\hline Comunidad del Caribe (CARICOM) & 17,5 & 4,0 & $-9,7$ & $-34,2$ & $-35,2$ \\
\hline \multicolumn{6}{|c|}{$\begin{array}{l}\text { Fuente: Comisión Económica para América Latina y el Caribe (CEPAL), sobre la base de información de los bancos centrales, servicios de aduanas e } \\
\text { institutos de estadística de los países, y Fondo Monetario Internacional (FMI), Direction of Trade Statistics [base de datos en línea] https:// } \\
\text { data.imf.org/?sk=9D6028D4-F14A-464C-A2F2-59B2CD424B85 (para datos de Cuba, Panamá y la CARICOM). } \\
\text { Nota: Se estimaron los meses de abril y mayo para Cuba, los países de la CARICOM, Panamá y la República Dominicana. } \\
\text { a No incluye reexportaciones. } \\
\text { b Corresponde a los cuatro miembros. Los datos para Colombia y el Perú se presentan en Comunidad Andina. } \\
\text { c Excluye reexportaciones de la Zona Libre de Colón. }\end{array}$} \\
\hline
\end{tabular}

La mayor caída promedio del valor exportado tuvo lugar en la Comunidad Andina (23\%), debido al elevado peso de los productos energéticos y mineros en su canasta exportadora (63\% en promedio en 2018-2019). Entre diciembre de 2019 y mayo de 2020, los precios de todos los productos energéticos tuvieron reducciones de dos dígitos, y varios productos mineros también registraron disminuciones de sus precios.

- El valor de las exportaciones de bienes de México disminuyó un $20,8 \%$ entre enero y mayo de 2020, debido, sobre todo, al desplome de los envíos manufactureros $(20,9 \%)$, que representaron el $88 \%$ del valor exportado en ese período. Solo el sector agropecuario y las actividades extractivas diferentes a la petrolera mostraron leves aumentos del $3,5 \%$ y el $5,2 \%$, respectivamente. La contracción de los envíos se fue acentuando durante ese período, llegando a una caída interanual del $57 \%$ en mayo, producto de la fuerte recesión en los Estados Unidos. En mayo, el valor de las exportaciones automotrices cayó a una tasa anualizada del $90 \%$, mientras que los envíos de las industrias electrónica, de plásticos y de maquinaria y equipos se contrajeron un $52 \%$, un $51 \%$ y un $42 \%$, respectivamente (INEGI, 2020). 
- El valor de las exportaciones del Mercado Común del Sur (MERCOSUR) sufrió una reducción del $\mathbf{1 2 , 4 \%}$ entre enero y mayo de 2020 . Las mayores caídas se registraron en la República Bolivariana de Venezuela $(65 \%)$ y el Uruguay $(21,4 \%)$. En el primer caso, esto se explica en gran medida por la marcada disminución del precio del petróleo. En el Uruguay, la mayor parte del impacto negativo obedece a la merma de las ventas de manufacturas industriales y agropecuarias, con reducciones superiores al $80 \%$ en productos como muebles, hierro y acero y vehículos, que se comercializan sobre todo en el MERCOSUR (CIU, 2020). En la Argentina y el Brasil, las mayores bajas se produjeron en los envíos de manufacturas (vehículos, autopartes, productos químicos), afectados por la menor demanda intrarregional, y en los de combustibles. Por el contrario, en ambos países aumentaron los volúmenes exportados de productos agropecuarios (carnes, lácteos, soja, frutas, azúcar), principalmente a China y otros países de Asia (INDEC, 2020; Ministerio de Economía del Brasil, 2020).

Solo cuatro países de la región, todos centroamericanos, aumentaron sus exportaciones de bienes entre enero y mayo de 2020. Costa Rica se vio beneficiada por el aumento de la demanda de dispositivos médicos para enfrentar la pandemia, especialmente en los Estados Unidos. Por su parte, Guatemala y Honduras aumentaron sus exportaciones de equipos de protección personal, especialmente mascarillas, y de productos agrícolas. Nicaragua se benefició del alza del precio del oro y de los volúmenes exportados de productos agrícolas y agropecuarios (café, caña de azúcar, frijoles, tabaco, entre otros). Parte de la resiliencia económica de estos países se explicaría por la importancia de los intercambios dentro de la propia subregión, que contribuyen a amortiguar la menor demanda en sus socios extrarregionales. Centroamérica exhibe el mayor coeficiente de comercio intrarregional en América Latina y el Caribe, que en 2018 alcanzó el 29,8\% (CEIE, 2019).

Entre enero y mayo se registraron fuertes caídas en el valor de los envíos de América Latina y el Caribe a los Estados Unidos, la Unión Europea y la propia región, que conjuntamente absorbieron el $69 \%$ de sus exportaciones totales de bienes en 2019. Por el contrario, los envíos a Asia han mostrado una mayor resiliencia. En particular, las exportaciones a China cayeron menos del $\mathbf{2} \%$ entre enero y mayo, y se recuperaron en abril y mayo, lo que coincide con la gradual reapertura de su economía (véase el cuadro 3 ).

Cuadro 3 | América Latina y el Caribe: variación interanual del valor de las exportaciones de bienes por destino, enero-mayo de 2018 a enero-mayo de 2020, y abril y mayo de 2020 en comparación con igual mes del año anterior (En porcentajes)

\begin{tabular}{lccccc}
\hline Destino & $\begin{array}{c}\text { Enero-mayo } \\
\mathbf{2 0 1 8}\end{array}$ & $\begin{array}{c}\text { Enero-mayo } \\
\mathbf{2 0 1 9}\end{array}$ & $\begin{array}{c}\text { Enero-mayo } \\
\mathbf{2 0 2 0}\end{array}$ & Abril 2020 & Mayo 2020 \\
\hline Mundo & $\mathbf{1 0 , 7}$ & $\mathbf{- 0 , 3}$ & $-16,6$ & $\mathbf{- 2 9 , 5}$ & $\mathbf{- 3 7 , 1}$ \\
\hline Estados Unidos & 6,6 & $\mathbf{4}, 9$ & $-22,2$ & $-40,5$ & $-56,0$ \\
\hline Unión Europea & 18,7 & $-4,5$ & $-14,3$ & $-22,8$ & $-31,2$ \\
\hline Asia & 14,9 & 2,4 & $-4,2$ & $-1,3$ & $-6,1$ \\
\hline China & 19,0 & 0,2 & $-1,2$ & 3,8 & 3,8 \\
\hline Resto de Asia & 10,3 & 5,1 & $-6,6$ & $-11,3$ & $-22,0$ \\
\hline América Latina y el Caribe & 12,5 & $-9,8$ & $-23,9$ & $-38,8$ & $-37,4$ \\
\hline
\end{tabular}

Fuente: Comisión Económica para América Latina y el Caribe (CEPAL), sobre la base de información de los bancos centrales, servicios de aduanas e institutos de estadística de los países.

El valor de los envíos dirigidos a la propia región tuvo una caída superior al promedio entre enero y mayo de 2020 (24\%), debido a la debilidad de la demanda en un contexto marcadamente recesivo (véase el gráfico 4). La contracción del comercio intrarregional se ha manifestado especialmente en las manufacturas. El sector más golpeado ha sido el automotor, con una caída cercana al $55 \%$ del valor de los intercambios entre enero y mayo de 2020 , seguido del sector de textiles, confecciones y calzado. Solo el comercio de productos agroindustriales registró una modesta expansión (véase el gráfico 5). 
Gráfico 4 | América Latina y el Caribe: variación interanual del valor de las exportaciones intrarregionales de bienes, enero de 2007 a mayo de 2020

(En porcentajes)

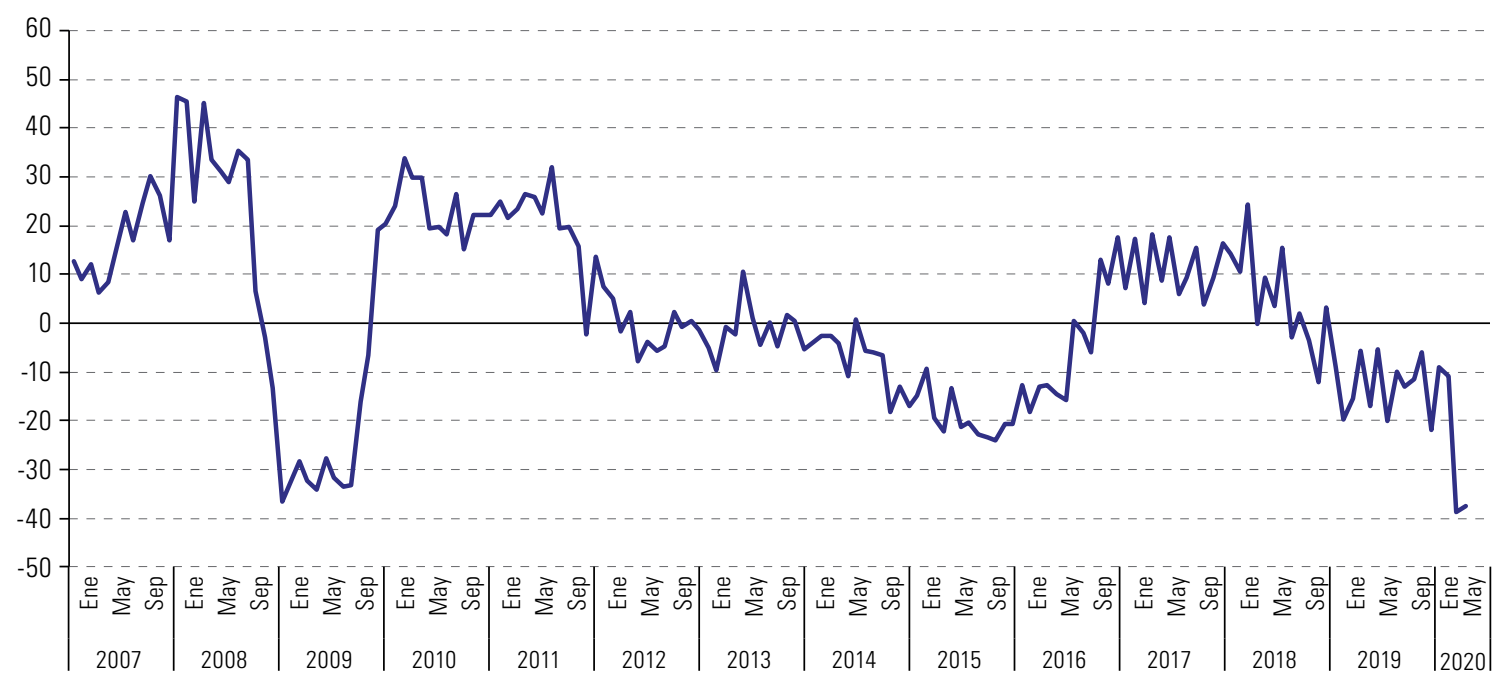

Fuente: Comisión Económica para América Latina y el Caribe (CEPAL), sobre la base de información de los bancos centrales, servicios de aduanas e institutos de estadística de los países.

Gráfico 5 | América Latina y el Caribe: variación del valor de las exportaciones intrarregionales por sector, enero-mayo de 2020 en comparación con igual período de 2019 (En porcentajes)

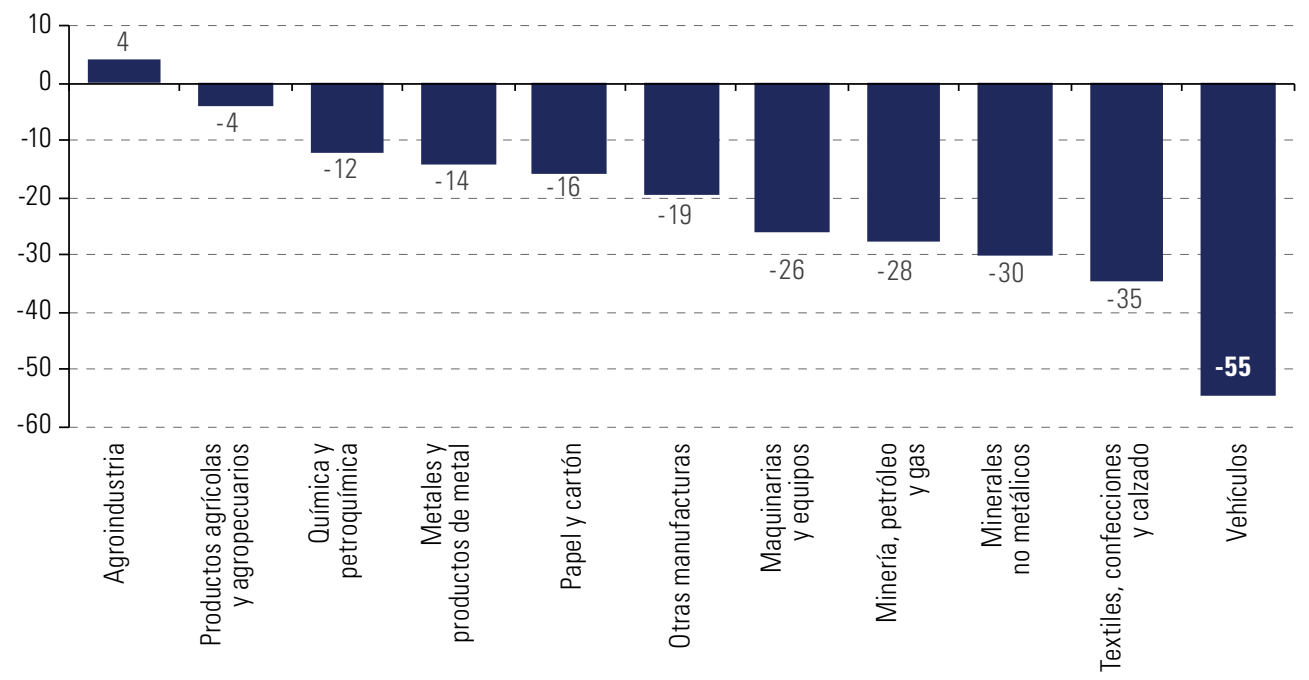

Fuente: Comisión Económica para América Latina y el Caribe (CEPAL), sobre la base de información de los bancos centrales, servicios de aduanas e institutos de estadística de los países.

La contracción del comercio intrarregional se dio en todos los principales bloques de integración económica. Entre enero y mayo de 2020, el valor de los intercambios en casi todos ellos registró disminuciones interanuales de entre el $20 \%$ y el $31 \%$. La única excepción fue el comercio entre los miembros del Mercado Común Centroamericano (MCCA), que mostró una mayor resiliencia al caer un 5,6\% (véase el cuadro 4). 
Cuadro 4 | América Latina y el Caribe: tasa de variación interanual de las exportaciones intrarregionales en los diversos bloques de integración, enero-mayo de 2019 y enero-mayo de 2020

(En porcentajes)

\begin{tabular}{|c|c|c|c|c|}
\hline \multirow{2}{*}{$\begin{array}{l}\text { Región o bloque } \\
\text { de integración }\end{array}$} & \multicolumn{2}{|c|}{ Tasa de variación } & \multicolumn{2}{|c|}{$\begin{array}{c}\text { Coeficiente de comercio intrarregional } \\
\text { medido por exportaciones }\end{array}$} \\
\hline & Enero-mayo 2019 & Enero-mayo 2020a & Enero-mayo 2019 & Enero-mayo 2020 \\
\hline América Latina y el Caribe & $-9,8$ & $-23,9$ & 13,7 & 12,5 \\
\hline $\begin{array}{l}\text { Mercado Común del Sur } \\
\text { (MERCOSUR) }\end{array}$ & $-19,7$ & $-22,8$ & 11,0 & 9,0 \\
\hline Comunidad Andina (CAN) & $-2,6$ & $-28,9$ & 7,3 & 7,3 \\
\hline $\begin{array}{l}\text { Mercado Común } \\
\text { Centroamericano (MCCA) }\end{array}$ & $-1,5$ & $-5,6$ & 27,4 & 24,7 \\
\hline Alianza del Pacífico & $-3,2$ & $-19,9$ & 2,8 & 2,7 \\
\hline $\begin{array}{l}\text { Comunidad del Caribe } \\
\text { (CARICOM)(enero-abril) }\end{array}$ & $\ldots$ & $-31,2$ & 11,4 & 7,4 \\
\hline
\end{tabular}

Fuente: Comisión Económica para América Latina y el Caribe (CEPAL), sobre la base de información de los bancos centrales, servicios de aduanas e institutos de estadística de los países, y Fondo Monetario Internacional (FMI), Direction of Trade Statistics [base de datos en línea] https://data.imf.org/?sk=9D6028D4-F14A-464C-A2F2-59B2CD424B85 (para datos de la CARICOM).

${ }^{\text {a }}$ Cifras preliminares.

\section{Dinámica de las exportaciones por sectores}

- Entre enero y mayo, el valor de los envíos regionales de productos mineros y petróleo y de manufacturas se desplomó un $\mathbf{2 5 , 8 \%}$ y un $\mathbf{1 8 , 5} \%$, respectivamente, en comparación con igual período de 2019. Por el contrario, las exportaciones agrícolas y agropecuarias aumentaron un $\mathbf{0 , 9} \%$ (véanse el cuadro 5 y el gráfico 6). Ello refleja la menor sensibilidad de la demanda de alimentos a la contracción de la actividad económica, al tratarse de bienes esenciales.

- En el caso de los productos mineros y el petróleo, la contracción del valor exportado obedece a los menores volúmenes exportados (consecuencia de la menor demanda) y las caídas significativas de sus precios. En las manufacturas, la disminución es atribuible principalmente a los menores volúmenes comercializados debido a choques de oferta (la paralización de industrias como la automotriz) y de demanda (la recesión en los principales mercados de esos productos).

En el caso de los productos agrícolas y agropecuarios, la Argentina, el Brasil, el Paraguay y el Uruguay se vieron favorecidos por la disminución de las ventas de Australia a China, principalmente a causa de la sequía en ese país, que redujo la producción de granos. Los países del MERCOSUR también se vieron beneficiados por el aumento de las importaciones de carne vacuna y porcina en China para asegurar el abastecimiento de alimentos.

El índice de precios de los productos básicos (que incluye productos que representaron casi el $40 \%$ del valor de las exportaciones totales de bienes de la región en 2018 y 2019) sufrió un desplome del 18,2\% en enero-junio de 2020 (véase el cuadro 6). La caída fue mucho mayor en los productos del rubro energía (37\%), especialmente el petróleo y sus derivados.

Cuadro 5 | América Latina y el Caribe: variación interanual del valor de las exportaciones de bienes según grandes sectores, enero-mayo de 2018 a enero-mayo de 2020, y abril y mayo de 2020 en comparación con igual mes del año anterior (En porcentajes)

\begin{tabular}{lcccccc}
\hline Sector & $\begin{array}{c}\text { Participación } \\
\text { en el total } \\
(\mathbf{2 0 1 9 )}\end{array}$ & $\begin{array}{c}\text { Enero-mayo } \\
\mathbf{2 0 1 8}\end{array}$ & $\begin{array}{c}\text { Enero-mayo } \\
\mathbf{2 0 1 9}\end{array}$ & $\begin{array}{c}\text { Enero-mayo } \\
\mathbf{2 0 2 0}\end{array}$ & Abril & Mayo \\
\hline Todos los sectores & $\mathbf{1 0 0 , 0}$ & $\mathbf{1 0 , 7}$ & $-\mathbf{0 , 3}$ & $\mathbf{- 1 6 , 6}$ & $\mathbf{- 2 9 , 5}$ & $\mathbf{- 3 7 , 1}$ \\
\hline $\begin{array}{l}\text { Productos agrícolas } \\
\text { y agropecuarios }\end{array}$ & 13,4 & 3,8 & 2,7 & 0,9 & $-5,2$ & $-4,2$ \\
\hline $\begin{array}{l}\text { Minería y petróleo } \\
\text { Manufacturas }\end{array}$ & 20,8 & 17,5 & $-5,1$ & $-25,8$ & $-41,6$ & $-43,0$ \\
\hline
\end{tabular}

Fuente: Comisión Económica para América Latina y el Caribe (CEPAL), sobre la base de información de los bancos centrales, servicios de aduanas e institutos de estadística de los países. 
Gráfico 6 | América Latina y el Caribe: variación interanual del valor de las exportaciones de bienes por grandes sectores, enero de 2007 a mayo de 2020

(En porcentajes)

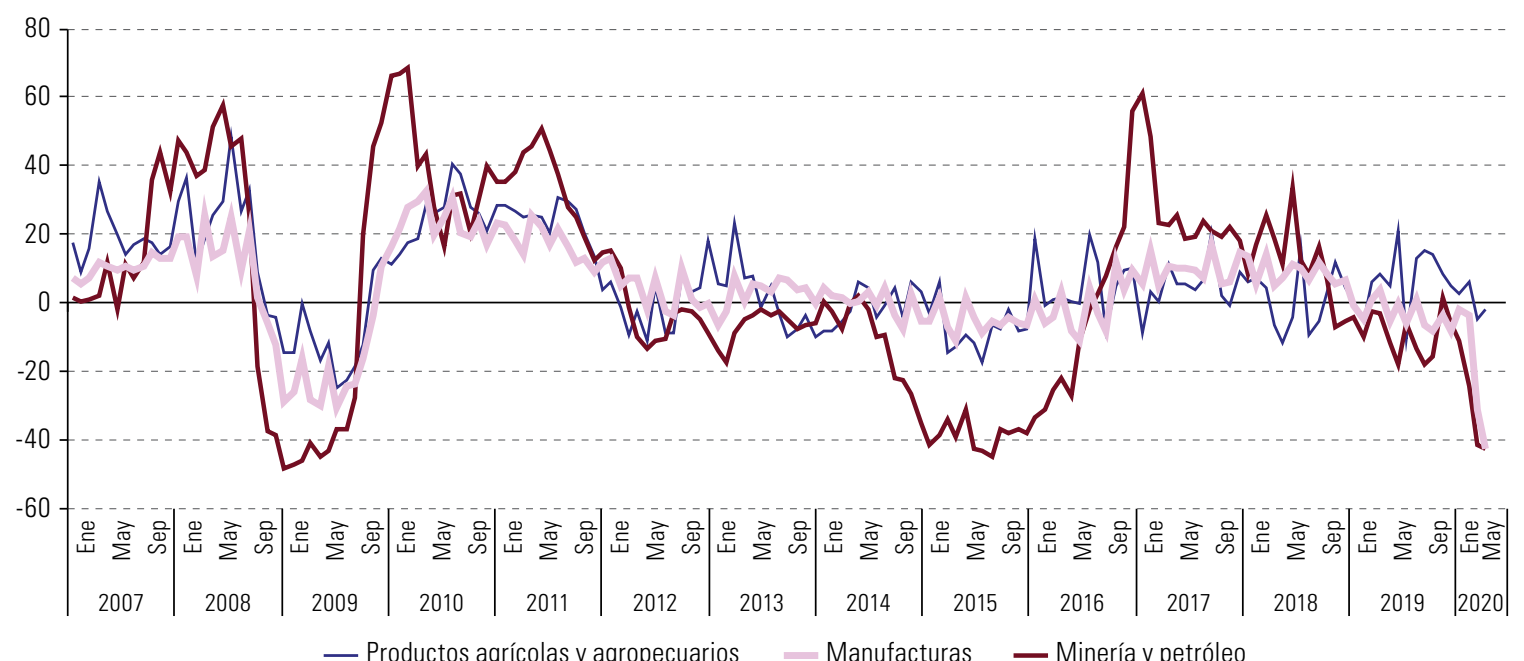

Fuente: Comisión Económica para América Latina y el Caribe (CEPAL), sobre la base de información de los bancos centrales, servicios de aduanas e institutos de estadística de los países.

Cuadro 6 | América Latina y el Caribe: variación de los precios de los principales productos básicos de exportación, y variación proyectada para 2020

(En porcentajes)

\begin{tabular}{|c|c|c|c|}
\hline & $\begin{array}{l}\text { Participación en las } \\
\text { exportaciones totales de bienes }\end{array}$ & $\begin{array}{l}\text { Variación diciembre de } \\
2019 \text { a junio de } 2020\end{array}$ & $\begin{array}{c}\text { Variación proyectada } \\
\text { en } 2020\end{array}$ \\
\hline Total de productos básicos & 39,4 & $-18,2$ & $-18,5$ \\
\hline Energía & 14,7 & $-37,0$ & $-39,3$ \\
\hline Petróleo & 11,2 & $-37,5$ & $-40,2$ \\
\hline Derivados de petróleo & 2,0 & $-48,0$ & $-42,7$ \\
\hline Gas natural & 1,0 & $-22,7$ & $-30,7$ \\
\hline Carbón & 0,5 & $-11,5$ & $-21,8$ \\
\hline Minerales y metales & 9,4 & $-1,9$ & $-6,1$ \\
\hline Otros minerales y metales & 2,6 & $-13,4$ & $-16,2$ \\
\hline Cobre & 2,5 & $-7,0$ & $-12,6$ \\
\hline Oro & 2,0 & 14,9 & 15,9 \\
\hline Hierro & 1,3 & 10,1 & $-9,5$ \\
\hline Aluminio & 0,5 & $-10,9$ & $-14,8$ \\
\hline Níquel & 0,1 & $-22,5$ & $-17,7$ \\
\hline Estaño & 0,1 & $-3,3$ & $-14,5$ \\
\hline Agrícolas y agropecuarios & 15,3 & $-10,1$ & $-5,9$ \\
\hline Aceite de soja & 2,1 & $-21,2$ & $-7,8$ \\
\hline Banano & 2,4 & 13,0 & 11,2 \\
\hline Carne bovina & 2,1 & $-15,5$ & $-5,1$ \\
\hline Soja & 1,9 & $-11,1$ & $-9,3$ \\
\hline Café & 1,3 & $-19,6$ & $-9,2$ \\
\hline Azúcar & 1,2 & $-22,0$ & $-11,4$ \\
\hline Harina de pescado & 1,0 & 1,3 & $-5,1$ \\
\hline Maíz & 1,0 & 1,8 & $-5,5$ \\
\hline Camarones y crustáceos & 0,7 & $-6,1$ & 4,3 \\
\hline
\end{tabular}

Fuente: Comisión Económica para América Latina y el Caribe (CEPAL), sobre la base de datos de Bloomberg, The Economist Intelligence Unit, la Bolsa de Cereales de Buenos Aires, la Corporación Chilena del Cobre (COCHILCO) y la Oficina de Estudios y Políticas Agrarias de Chile (ODEPA). 
- El precio del petróleo registró mínimos históricos durante abril. El barril de West Texas Intermediate (WTI) llegó a cotizarse en valores negativos en el mercado de futuros, en el contexto de una marcada reducción de la demanda global y una guerra de precios entre algunos de los principales productores. Después de una disminución de hasta el $66 \%$ en abril-mayo, que lo mantuvo en torno a 30 dólares el barril, el precio ha repuntado levemente y se mantiene volátil.

- Los precios de los minerales y metales también han sufrido las consecuencias de las menores expectativas de demanda global. Hasta junio, la mayoría de ellos anotó mermas en sus precios de entre el $10 \%$ y el $17 \%$. Su evolución futura depende fundamentalmente de la reactivación de la producción siderúrgica y metalmecánica, y de la construcción en China. En el primer semestre, solo aumentaron el precio del oro y el mineral de hierro. En el caso del primero, debido principalmente a su calidad de reserva de valor. En el del segundo, el aumento de su precio desde la segunda mitad de mayo obedece a un brote de la pandemia en el complejo Itabira de la empresa Vale. Dado que el $70 \%$ de la oferta del mineral transportado por vía marítima corresponde al Brasil, el impacto fue inmediato durante junio, con un alza del $10 \%$ con respecto al cierre de su cotización en diciembre. Se espera que el precio vuelva a bajar cuando se restablezcan las operaciones.

Entre los productos agrícolas y agropecuarios, las reducciones de los precios han sido menores que el promedio del resto de los productos básicos, destacándose las alzas en el precio del banano y de los camarones y crustáceos.

\section{Dinámica de las importaciones}

Entre enero y mayo de 2020, el valor de las importaciones de bienes de la región cayó un $17,1 \%$, debido a una baja del volumen del $12 \%$ y una reducción de los precios del $5 \%$. Las compras externas disminuyeron en todas las categorías de bienes, contracción que se acentuó a lo largo de ese período (véanse el cuadro 7 y gráfico 7). La mayor caída se produjo en los combustibles (34\%), resultado influido por la disminución de sus precios. Todas las otras categorías tuvieron descensos superiores al 10\%. Particularmente preocupante resulta la contracción de las importaciones de bienes de capital y de insumos intermedios $(14,5 \%$ y $13,6 \%$, respectivamente), que afectará la tasa de inversión y comprometerá la recuperación.

- El valor de las importaciones se contrajo en todos los países de la región (véase el cuadro 8), como resultado de la recesión. A diferencia de lo ocurrido con las exportaciones, las importaciones se contrajeron marcadamente desde todos los principales países proveedores (véase el cuadro 9).

Cuadro 7 | América Latina y el Caribe: variación interanual del valor de las importaciones de bienes según grandes categorías económicas, enero-mayo de 2018 a enero-mayo de 2020, y abril y mayo de 2020 en comparación con igual período del año anterior (En porcentajes)

\begin{tabular}{lcccccc}
\hline Categorias & $\begin{array}{c}\text { Participación } \\
\text { en el total } \\
(\mathbf{2 0 1 9 )}\end{array}$ & $\begin{array}{c}\text { Enero-mayo } \\
\mathbf{2 0 1 8}\end{array}$ & $\begin{array}{c}\text { Enero-mayo } \\
\mathbf{2 0 1 9}\end{array}$ & $\begin{array}{c}\text { Enero-mayo } \\
\mathbf{2 0 2 0}\end{array}$ & Abril & Mayo \\
\hline Todas las categorías & $\mathbf{1 0 0 , 0}$ & $\mathbf{1 3 , 5}$ & $\mathbf{- 1 , 3}$ & $\mathbf{- 1 7 , 1}$ & $\mathbf{- 2 8 , 0}$ & $\mathbf{- 3 7 , 0}$ \\
\hline Bienes de capital & 14,6 & 17,1 & $-5,2$ & $-14,5$ & $-29,4$ & $-22,9$ \\
\hline Insumos intermedios & 54,8 & 10,1 & 0,9 & $-13,6$ & $-16,9$ & $-37,0$ \\
\hline Bienes de consumo & 18,2 & 11,7 & $-3,9$ & $-17,1$ & $-31,3$ & $-39,1$ \\
\hline Combustibles & 12,5 & 33,0 & 4,7 & $-34,0$ & $-59,2$ & $-63,7$ \\
\hline
\end{tabular}

Fuente: Comisión Económica para América Latina y el Caribe (CEPAL), sobre la base de información de los bancos centrales, servicios de aduanas e institutos de estadística de los países. 
Gráfico 7 | América Latina: variación interanual del valor de las importaciones de bienes según grandes categorías económicas, enero de 2007 a mayo de 2020

(En porcentajes)

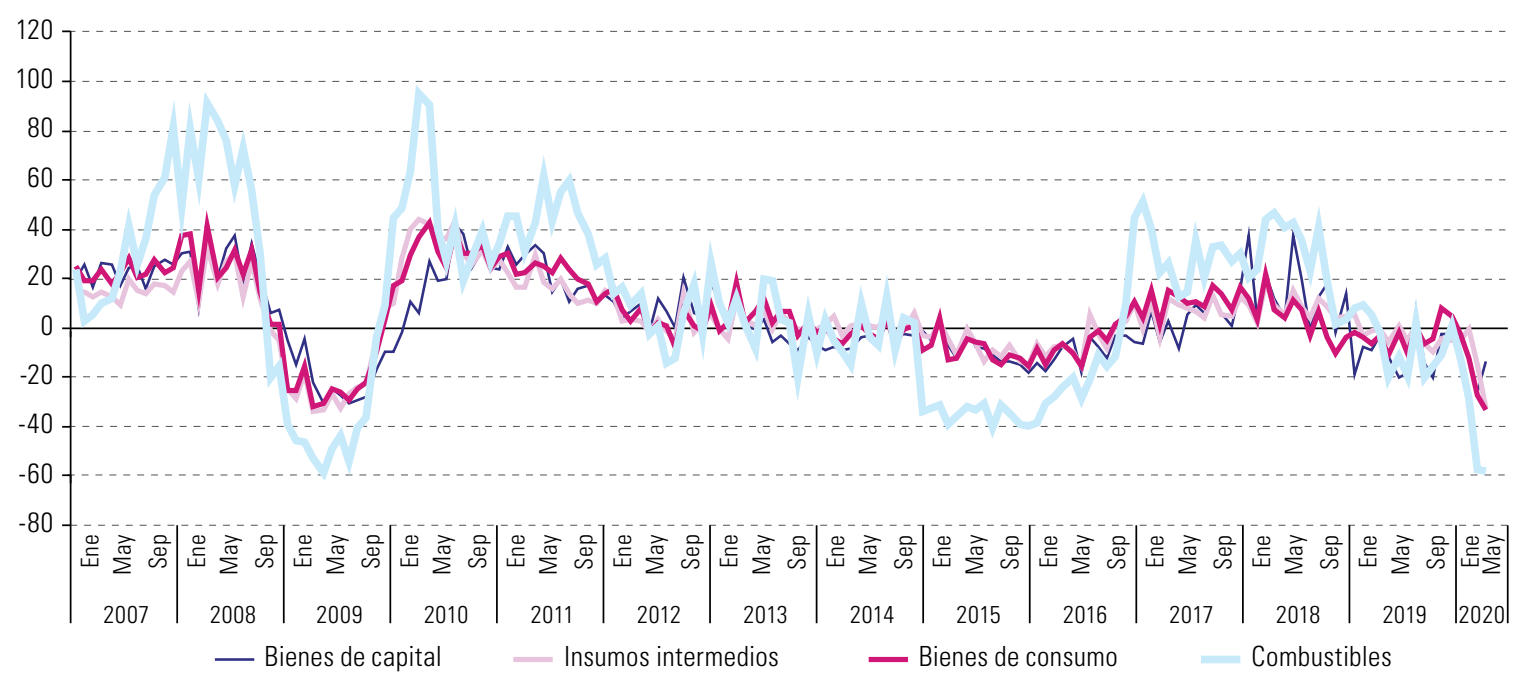

Fuente: Comisión Económica para América Latina y el Caribe (CEPAL), sobre la base de información de los bancos centrales, servicios de aduanas e institutos de estadística de los países.

Cuadro 8 | América Latina y el Caribe: variación interanual del valor de las importaciones de bienes, enero-mayo de 2018 a enero-mayo de 2020, y abril y mayo de 2020 en comparación con el mismo período del año anterior (En porcentajes)

\begin{tabular}{|c|c|c|c|c|c|}
\hline Región/Subregión/País & $\begin{array}{c}\text { Enero-mayo } \\
2018 \\
\end{array}$ & $\begin{array}{c}\text { Enero-mayo } \\
2019 \\
\end{array}$ & $\begin{array}{c}\text { Enero-mayo } \\
2020 \\
\end{array}$ & Abril 2020 & Mayo 2020 \\
\hline América Latina y el Caribe & 13,5 & $-1,3$ & $-17,1$ & $-28,0$ & $-37,0$ \\
\hline MERCOSUR & 18,3 & $-10,1$ & $-8,9$ & $-20,3$ & $-17,1$ \\
\hline Argentina & 18,8 & $-28,7$ & $-23,8$ & $-30,1$ & $-31,8$ \\
\hline Brasil & 18,0 & 1,8 & $-2,5$ & $-14,8$ & $-10,5$ \\
\hline Paraguay & 20,1 & $-7,2$ & $-19,9$ & $-50,7$ & $-36,1$ \\
\hline Uruguay & 12,5 & $-10,1$ & $-6,3$ & $-18,8$ & $-17,5$ \\
\hline Venezuela (República Bolivariana de) & 22,0 & $-56,8$ & $-45,1$ & $-36,3$ & $-42,0$ \\
\hline Comunidad Andina & 11,3 & 3,2 & $-20,5$ & $-34,4$ & $-42,9$ \\
\hline Bolivia (Estado Plurinacional de) & 5,2 & 7,3 & $-37,7$ & $-63,3$ & $-61,0$ \\
\hline Colombia & 6,0 & 5,2 & $-17,8$ & $-28,4$ & $-45,4$ \\
\hline Ecuador & 23,1 & 4,5 & $-25,2$ & $-41,3$ & $-48,9$ \\
\hline Perú & 13,4 & $-0,7$ & $-18,5$ & $-31,8$ & $-36,9$ \\
\hline Alianza del Pacífico & 12,4 & 1,7 & $-19,3$ & $-29,6$ & $-44,6$ \\
\hline Chile & 16,9 & $-0,5$ & $-20,5$ & $-22,7$ & $-36,4$ \\
\hline México & 12,3 & 1,9 & $-19,0$ & $-30,5$ & $-47,1$ \\
\hline Mercado Común Centroamericano & 9,0 & $-2,5$ & $-14,0$ & $-25,4$ & $-22,6$ \\
\hline Costa Rica & 6,0 & $-1,3$ & $-9,1$ & $-18,6$ & $-15,1$ \\
\hline El Salvador & 11,3 & 6,2 & $-18,0$ & $-32,9$ & $-45,2$ \\
\hline Guatemala & 10,0 & 1,9 & $-7,5$ & $-16,0$ & $-18,1$ \\
\hline Honduras & 10,6 & $-0,5$ & $-14,9$ & $-28,9$ & $-22,5$ \\
\hline Nicaragua & 4,9 & $-21,4$ & $-28,2$ & $-43,9$ & $-31,2$ \\
\hline Panamáa & 10,5 & $-11,0$ & $-25,7$ & $-32,0$ & $-37,4$ \\
\hline Países del Caribe & 7,7 & 7,1 & $-25,8$ & $-35,9$ & $-44,8$ \\
\hline Cuba & 6,9 & $-2,1$ & $-40,7$ & $-57,1$ & $-41,3$ \\
\hline República Dominicana & 11,6 & 4,7 & $-21,5$ & $-29,9$ & $-48,5$ \\
\hline Comunidad del Caribe (CARICOM) & 5,5 & 11,0 & $-25,1$ & $-33,8$ & $-43,1$ \\
\hline
\end{tabular}

Fuente: Comisión Económica para América Latina y el Caribe (CEPAL), sobre la base de información de los bancos centrales, servicios de aduanas e institutos de estadística de los países, y Fondo Monetario Internacional (FMI), Direction of Trade Statistics [base de datos en línea] https:// data.imf.org/?sk=9D6028D4-F14A-464C-A2F2-59B2CD424B85 (para datos de Cuba, la República Dominicana y la CARICOM).

Nota: Se estimaron los meses de abril y mayo para Cuba, los países de la CARICOM, Panamá y la República Dominicana.

a No incluye información de la Zona Libre Colón. 
Cuadro 9 | América Latina y el Caribe: variación interanual del valor de las importaciones de bienes por origen, enero-mayo de 2018 a enero-mayo de 2020, y abril y mayo de 2020 en comparación con el mismo mes del año anterior (En porcentajes)

\begin{tabular}{|c|c|c|c|c|c|}
\hline & $\begin{array}{c}\text { Enero-mayo } \\
2018 \\
\end{array}$ & $\begin{array}{c}\text { Enero-mayo } \\
2019\end{array}$ & $\begin{array}{c}\text { Enero-mayo } \\
2020 \\
\end{array}$ & Abril 2020 & Mayo 2020 \\
\hline Mundo & 13,5 & $-1,3$ & $-17,1$ & $-28,0$ & $-37,0$ \\
\hline Estados Unidos & 10,6 & $-0,5$ & $-19,6$ & $-38,2$ & $-48,7$ \\
\hline Unión Europea & 23,3 & $-5,3$ & $-18,1$ & $-23,1$ & $-39,0$ \\
\hline Asia & 13,4 & 5,2 & $-16,0$ & $-21,8$ & $-34,2$ \\
\hline China & 17,0 & 6,0 & $-17,4$ & $-21,4$ & $-33,4$ \\
\hline Resto de Asia & 9,0 & 4,1 & $-14,1$ & $-22,2$ & $-35,0$ \\
\hline América Latina y el Caribe & 11,8 & $-7,8$ & $-25,1$ & $-34,5$ & $-42,4$ \\
\hline
\end{tabular}

Fuente: Comisión Económica para América Latina y el Caribe (CEPAL), sobre la base de información de los bancos centrales, servicios de aduanas e institutos de estadística de los países.

\section{El colapso del turismo arrastra a las exportaciones de servicios}

En el primer trimestre de 2020, el valor de las exportaciones e importaciones de servicios de un grupo de 11 países de la región disminuyó un $10,2 \%$ y un $9 \%$, respectivamente, en comparación con el mismo período de 2019 (véase el cuadro 10) ${ }^{2}$. Al igual que lo ocurrido con el comercio de bienes, la tendencia del comercio de servicios fue declinante a lo largo del trimestre (véase el gráfico 8). Sin embargo, el desplome del valor del comercio de servicios en esos países fue muy superior al del comercio de bienes, que anotó caídas del $2,7 \%$ y el $4,6 \%$ en las exportaciones e importaciones, respectivamente.

- La mayor caída de las exportaciones regionales de servicios entre enero y marzo de 2020 se observa en el componente viajes $(\mathbf{1 7 , 4} \%)$, debido a la virtual paralización de la llegada de turistas internacionales (véase el gráfico 9). En segundo lugar, se ubican las exportaciones de servicios de transporte, que caen un $6,8 \%$ en el contexto de la contracción del comercio regional de bienes y de restricciones para contener la pandemia. Las exportaciones de otros servicios sufren la menor caída $(2,4 \%)$, e incluso en esta categoría algunos rubros aumentan. En efecto, las medidas de distanciamiento social han aumentado la demanda de servicios que pueden suministrarse en forma digital como la comunicación, las compras, la educación, la medicina y la entretención (OMC, 2020b).

Cuadro 10 América Latina (11 países)a : variación interanual del valor del comercio de servicios por sector, primer trimestre de 2019 y de 2020

(En porcentajes)

\begin{tabular}{|c|c|c|c|c|}
\hline \multirow[b]{2}{*}{ Sector } & \multicolumn{2}{|c|}{ Exportaciones } & \multicolumn{2}{|c|}{ Importaciones } \\
\hline & $\begin{array}{l}\text { Enero-marzo } \\
2019\end{array}$ & $\begin{array}{l}\text { Enero-marzo } \\
2020\end{array}$ & $\begin{array}{l}\text { Enero-marzo } \\
2019\end{array}$ & $\begin{array}{c}\text { Enero-marzo } \\
2020\end{array}$ \\
\hline Total servicios & 0,0 & $-10,2$ & $-6,1$ & $-9,0$ \\
\hline 1. Transportes & 2,4 & $-6,8$ & $-3,3$ & $-7,5$ \\
\hline 2. Viajes & 1,5 & $-17,4$ & $-15,0$ & $-25,4$ \\
\hline 3. Otros servicios & $-3,0$ & $-2,4$ & 3,7 & $-0,1$ \\
\hline $\begin{array}{l}\text { Servicios de comunicaciones, } \\
\text { informática e información }\end{array}$ & 3,4 & 2,3 & $-2,5$ & 17,3 \\
\hline Servicios de construcción & 6,8 & $-1,6$ & $-55,3$ & $-39,0$ \\
\hline Servicios de seguros & 1,7 & 0,0 & 12,2 & $-0,4$ \\
\hline Servicios financieros & 6,4 & 24,3 & 19,6 & $-4,1$ \\
\hline Regalías y derechos de licencia & $-19,8$ & $-3,1$ & $-10,0$ & $-11,5$ \\
\hline Otros servicios empresariales & $-7,2$ & $-0,8$ & $-7,9$ & $-2,6$ \\
\hline $\begin{array}{l}\text { Servicios personales, culturales } \\
\text { y recreativos }\end{array}$ & 26,8 & 8,6 & $\ldots$ & 13,8 \\
\hline
\end{tabular}

Fuente: Comisión Económica para América Latina y el Caribe (CEPAL), sobre la base de información de los bancos centrales de los países.

a Los países considerados son: Argentina, Bolivia (Estado Plurinacional de), Brasil, Chile, Colombia, Costa Rica, El Salvador, México, Perú, República Dominicana y Uruguay. Estos países representan el $78 \%$ de las exportaciones y el 82\% de las importaciones de servicios comerciales de la región.

\footnotetext{
2 A diferencia de los bienes, la recopilación estadística sectorial para los servicios se realiza de modo trimestral, por lo que no se dispone de información para el período enero-mayo de 2020
} 
Gráfico 8 América Latina (11 países)a: variación interanual del valor del comercio de servicios, primer trimestre de 2007 a primer trimestre de 2020

(En porcentajes)

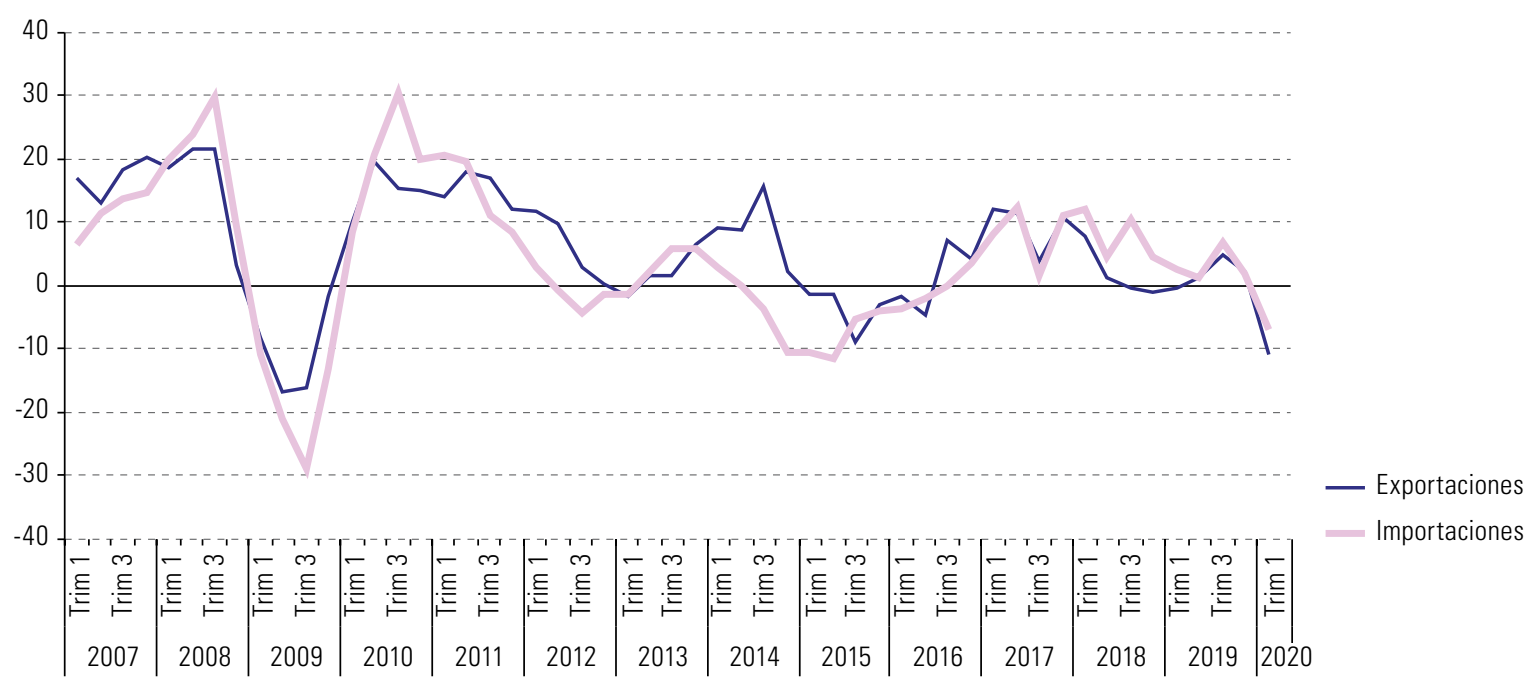

Fuente: Comisión Económica para América Latina y el Caribe (CEPAL), sobre la base de información de los bancos centrales de los países.

a Los países considerados son: Argentina, Bolivia (Estado Plurinacional de), Brasil, Chile, Colombia, Costa Rica, El Salvador, México, Perú, República Dominicana y Uruguay.

Gráfico 9 | América Latina y el Caribe (países seleccionados): variación interanual de las llegadas de turistas internacionales, enero de 2018 a mayo de 2020

(En porcentajes)

\section{A. Países de América del Sur}

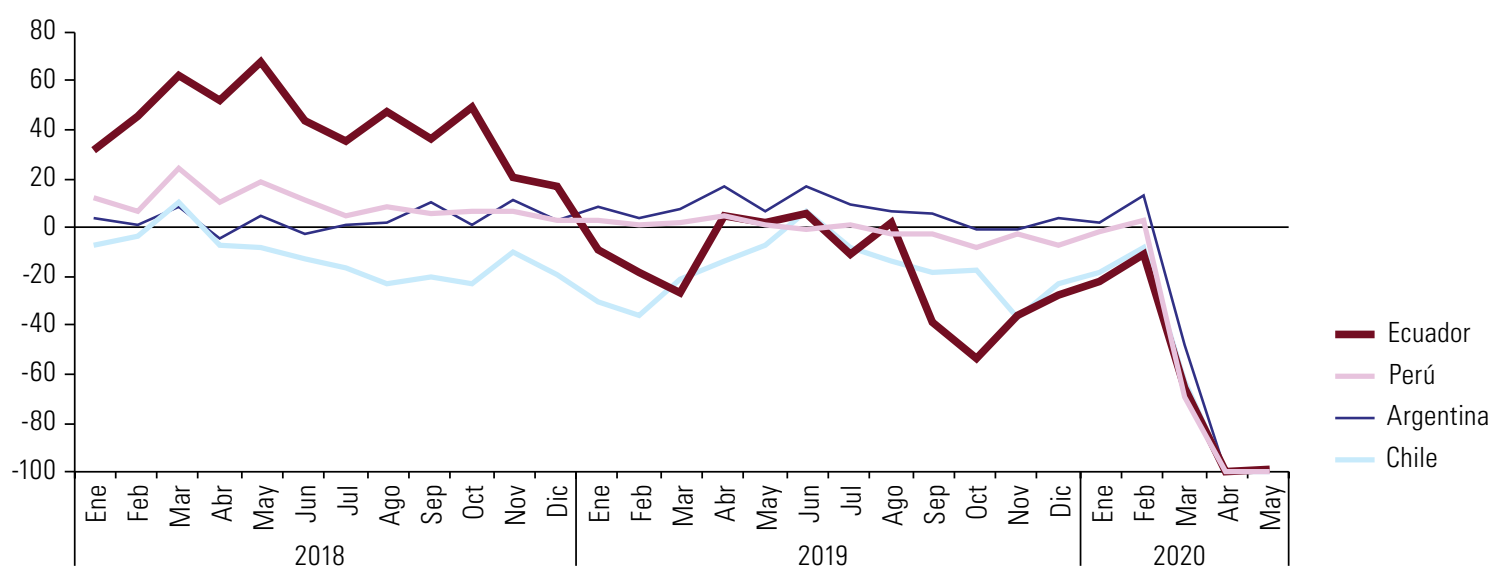

\section{B. Otros países}

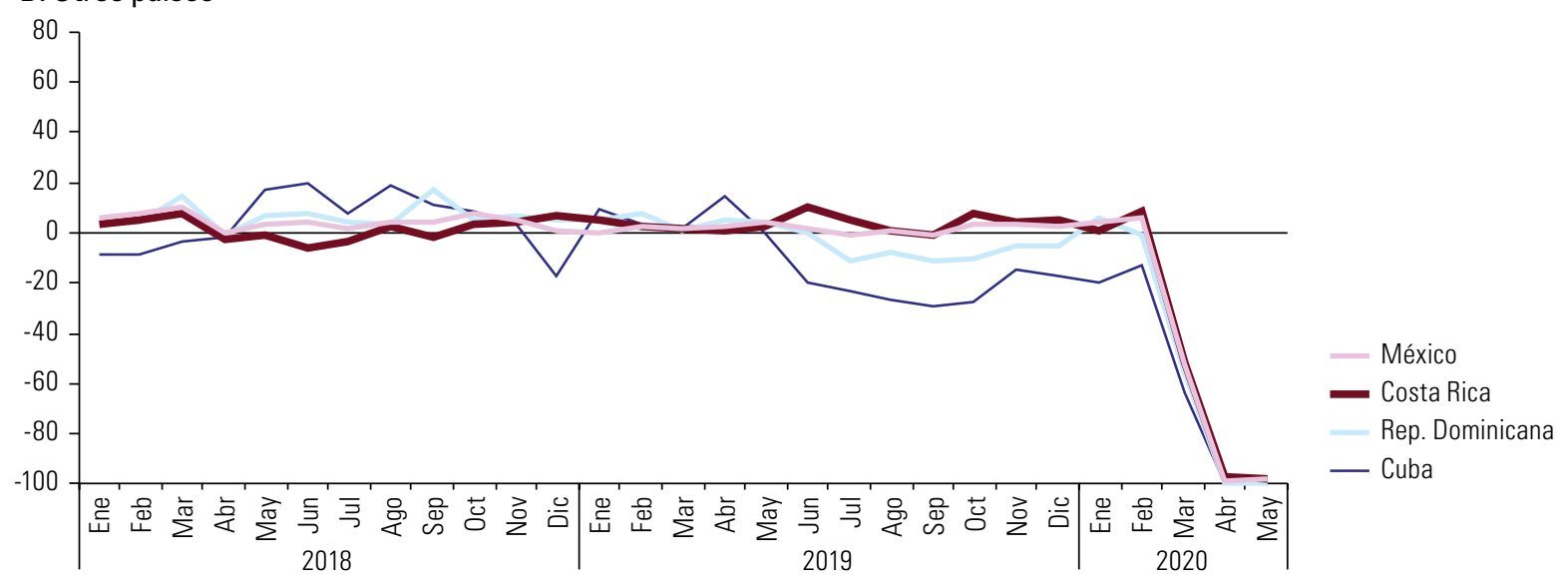

Fuente: Comisión Económica para América Latina y el Caribe (CEPAL), sobre la base de información oficial. 
La paralización del turismo es grave para la región pues representó el $\mathbf{4 8} \%$ del valor de sus exportaciones totales de servicios en 2019, el doble de su participación en las exportaciones mundiales de servicios. La situación es particularmente grave para los países del Caribe, en los cuales el turismo representó en 2019 el 45\% de las exportaciones totales de bienes y servicios. En este contexto, se proyecta que en 2020 el valor de las exportaciones de bienes y servicios del Caribe disminuya un $40 \%$ (véase el gráfico 10). Por su parte, el PIB y el empleo total podrían verse reducidos en alrededor de 8 y 9 puntos porcentuales, respectivamente (CEPAL, 2020b).

Gráfico 10 América Latina y el Caribe (países y subregiones seleccionados): caída proyectada de las exportaciones, el PIB y el empleo, 2020

A. Exportaciones de bienes y servicios (en porcentajes)

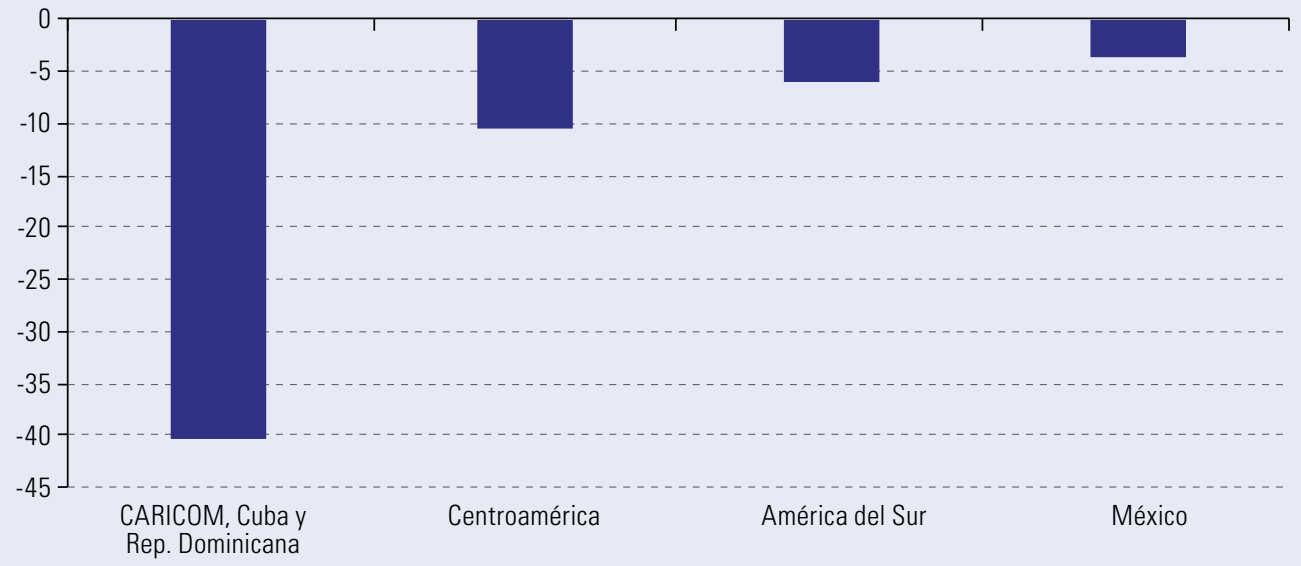

B. PIB y empleo (en puntos porcentuales)

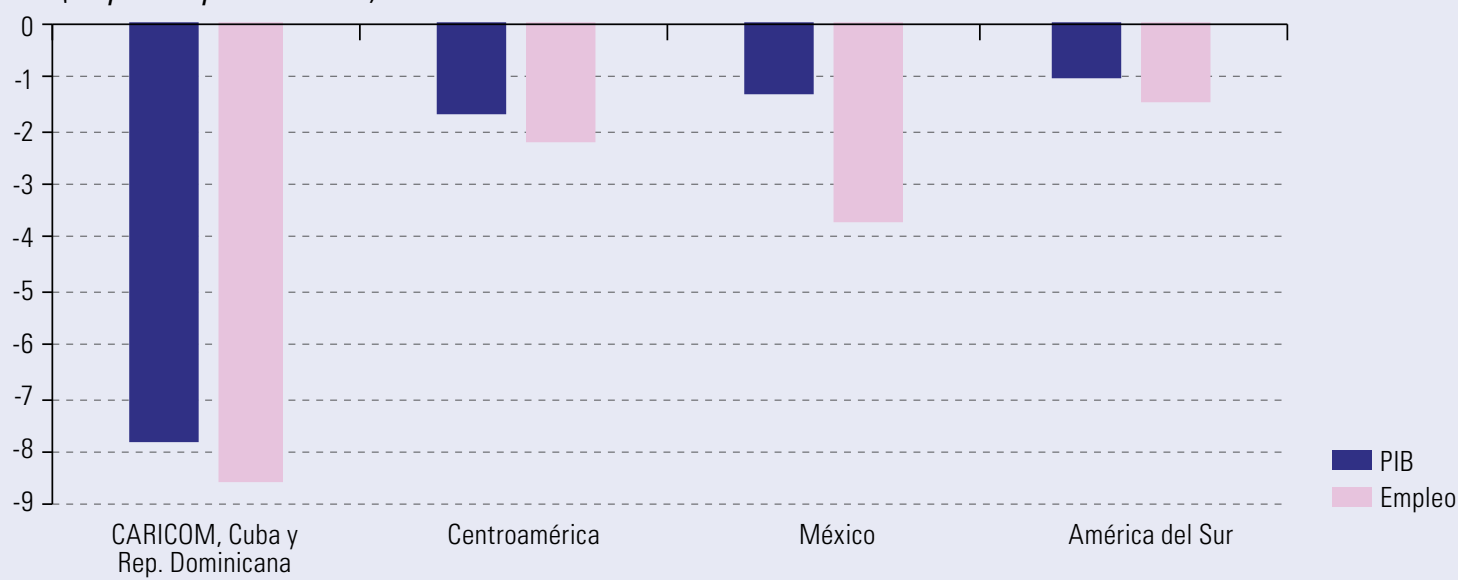

Fuente: Comisión Económica para América Latina y el Caribe (CEPAL), “Medidas de recuperación del sector turístico en América Latina y el Caribe: una oportunidad para promover la sostenibilidad y la resiliencia", Informes COVID-19, Santiago, julio de 2020.

Nota: En este análisis la Comunidad del Caribe (CARICOM) no incluye Montserrat; Centroamérica no incluye El Salvador y en el gráfico B no incluye Panamá; América del Sur no incluye la República Bolivariana de Venezuela.

\section{Los problemas en el transporte internacional traban} el comercio regional

- A nivel mundial, el comercio marítimo por contenedores ha seguido una tendencia descendente desde la irrupción de la pandemia. Aunque hasta febrero aumentaba en algunas regiones, desde marzo cayó en la gran mayoría de ellas (véase el cuadro 11). En América Latina, la variación interanual en el período enero-mayo de 2020 fue del $-6,1 \%$, con marcadas disminuciones en abril y mayo.

- A pesar de la caída del tráfico marítimo en 2020 , los fletes del transporte de contenedores se mantuvieron por encima de los valores de 2019 (véase el gráfico 11), salvo en el período de baja estacional anual debido al Año Nuevo chino. A fines de abril, se inició un alza sostenida de los fletes y 
el 2 de julio de 2020 superaban en un 48\% su nivel del año anterior. Ello sugiere que la industria pudo administrar la oferta y lograr así un nivel de precios que le permitió sortear en parte la disminución de la demanda causada por el COVID-19. Pese a ello, seis de las diez mayores navieras del mundo han solicitado ayuda de los gobiernos de sus países de origen: CMA CGM, COSCO Shipping Lines, HMM, Evergreen Marine, Yang Ming y Pacific International Lines (PIL).

Cuadro 11 | Variación interanual del comercio internacional marítimo por contenedores, enero a mayo de 2020 (En porcentajes)

\begin{tabular}{lcccccc}
\hline & Enero & Febrero & Marzo & Abril & Mayo & $\begin{array}{c}\text { Enero a } \\
\text { mayo }\end{array}$ \\
\hline América Latina & 3,1 & 2,9 & 2,5 & $-16,6$ & $-20,9$ & $-6,1$ \\
\hline América del Norte & $-0,5$ & $-6,8$ & $-3,2$ & $-15,5$ & $-17,3$ & $-8,8$ \\
\hline Europa & 0,9 & $-3,9$ & $-1,8$ & $-16,3$ & $-14,6$ & $-7,3$ \\
\hline $\begin{array}{l}\text { Lejano Oriente } \\
\begin{array}{l}\text { Subcontinente indio } \\
\text { y Oriente Medio }\end{array}\end{array}$ & $-12,4$ & $-6,1$ & $-13,8$ & $-6,0$ & $-7,0$ \\
\hline \begin{tabular}{l} 
Mundo \\
\hline
\end{tabular} & 3,5 & 6,5 & $-3,4$ & $-27,6$ & $-21,2$ & $-8,9$ \\
\hline
\end{tabular}

Fuente: Comisión Económica para América Latina y el Caribe (CEPAL), sobre la base de información de Container Trades Statistics (CTS).

Gráfico 11 | Costo de los fletes marítimos de contenedores, enero a junio de 2019 y enero a junio de 2020 (En dólares por contenedor de 40 pies)

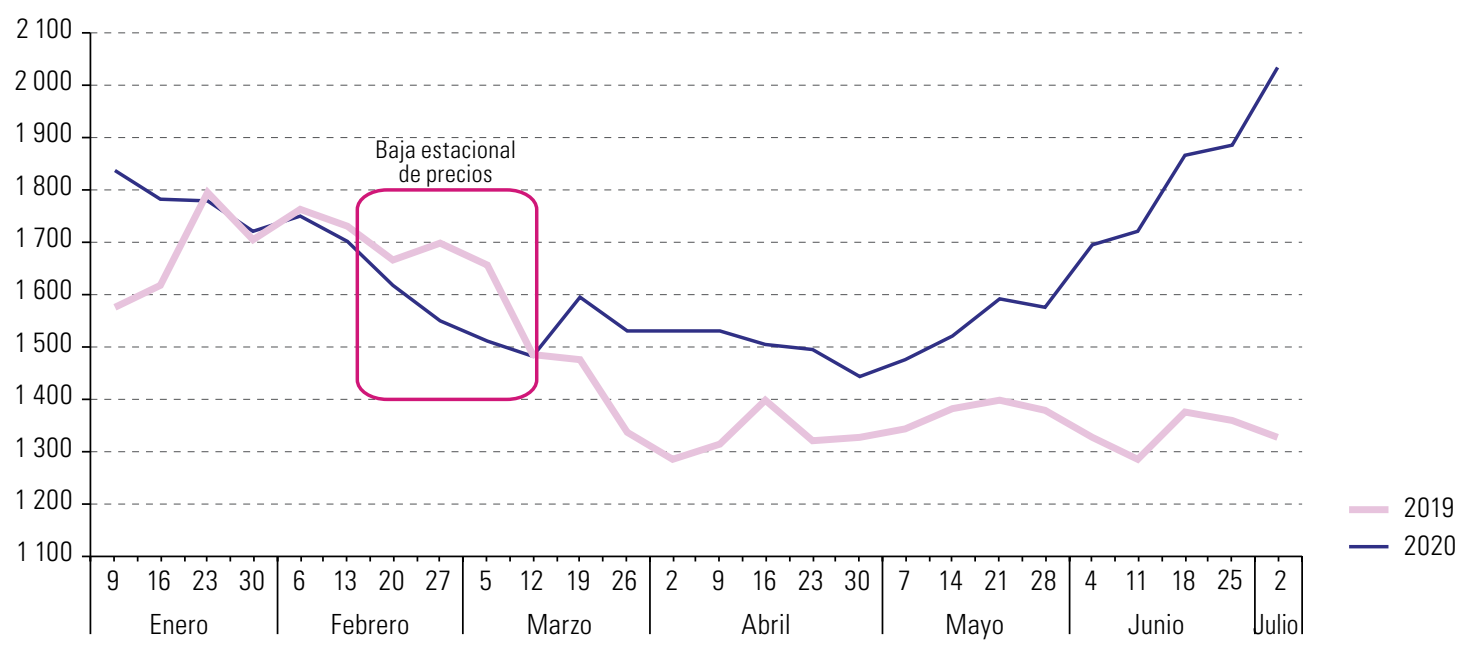

Fuente: Comisión Económica para América Latina y el Caribe (CEPAL), sobre la base de información de Drewry.

La irrupción de la pandemia ha causado una marcada reducción del movimiento portuario en gran parte de los puertos de la región. El cuadro 12 muestra la variación interanual de la actividad portuaria de contenedores entre enero y mayo de 2020 en una muestra de puertos que representa el $80 \%$ de la carga total de América Latina y el Caribe. La caída regional de la actividad portuaria de contenedores es de alrededor del $1 \%$, mientras que para el mismo período la suma de importaciones y exportaciones por contenedor se redujo un $6 \%$. La diferencia entre ambas cifras se explica porque otros movimientos portuarios, operativos y de transbordo compensaron la caída del comercio internacional en contenedores. Los datos preliminares recogidos al cierre de junio de 2020 permiten ver que en ese mes, tanto el movimiento total de contenedores como la suma del de importaciones y exportaciones por contenedor de la región se contrajo otros 4 puntos porcentuales respecto de la reducción observada hasta mayo.

- La creciente concentración de la industria naviera genera preocupación por su posible impacto en los fletes durante la contracción y la recuperación. En 1992, el mercado del transporte marítimo estaba compuesto principalmente por 30 empresas, que representaban alrededor del $63 \%$ de la flota total. En 1998, se conformaron seis alianzas, que representaban el $50 \%$ de la flota mundial. Entre 2000 y 2010, se duplicó la capacidad combinada de la flota de los 30 principales transportistas y alcanzó los 10,81 millones de unidades equivalentes a 20 pies (TEU). En 2018, tres de las alianzas tenían una participación combinada cercana al $70 \%$ de la oferta (capacidad) mundial, y en 2020 han aumentado su participación al 84,2\%. 
Cuadro 12 América Latina y el Caribe (puertos seleccionados): evolución de la actividad portuaria de contenedores, enero a mayo de 2020 en comparación con el mismo período de 2019

(En porcentajes)

\begin{tabular}{|c|c|c|c|c|}
\hline & $\begin{array}{l}\text { Puertos y porcentaje } \\
\text { de la actividad portuaria } \\
\text { de cada país }\end{array}$ & País & $\begin{array}{c}\text { Participación en el total } \\
\text { regional en unidades } \\
\text { equivalentes a } 20 \text { pies } \\
\text { (TEU)(2019) }\end{array}$ & $\begin{array}{c}\text { Variación } \\
\text { enero-mayo } 2020\end{array}$ \\
\hline \multicolumn{2}{|c|}{ Puertos con caídas de actividad } & \multicolumn{3}{|c|}{43,4} \\
\hline \multirow{13}{*}{$\frac{\infty}{\substack{0 \\
\frac{0}{0}}}$} & Buenaventura (25\%) & Colombia & 2,1 & $-32,9$ \\
\hline & Valparaíso (31\%) & Chile & 1,7 & $-28,0$ \\
\hline & Lázaro Cárdenas (19\%) & México & 2,4 & $-18,8$ \\
\hline & Freeport (85\%) & Bahamas & 2,6 & $-16,6$ \\
\hline & Caucedo (67\%) & República Dominicana & 2,3 & $-15,0$ \\
\hline & Altamira $(13 \%)$ & México & 1,6 & $-14,9$ \\
\hline & San Antonio (60\%) & Chile & 3,2 & $-10,2$ \\
\hline & Veracruz (16\%) & México & 2,1 & $-9,8$ \\
\hline & Río Grande (7\%) & Brasil & 1,4 & $-9,5$ \\
\hline & Brasil, otros $(28 \%)$ & Brasil & 5,3 & $-7,0$ \\
\hline & Iquique $(6,1 \%)$ & Chile & 0,2 & $-6,4$ \\
\hline & El Callao $(86 \%)$ & Perú & 4,3 & $-5,9$ \\
\hline & Manzanillo (42\%) & México & 5,7 & $-3,8$ \\
\hline \multirow{4}{*}{$\begin{array}{l}\infty \\
\frac{\pi}{0} \\
\frac{0}{0} \\
\frac{\pi}{0} \\
\frac{0}{0} \\
\sum\end{array}$} & Limón-Moin (80\%) & Costa Rica & 2,3 & $-2,3$ \\
\hline & Buenos Aires (84\%) & Argentina & 2,7 & $-1,5$ \\
\hline & Kingston (85\%) & Jamaica & 3,0 & $-1,1$ \\
\hline & Point Lisas (48\%) & Trinidad y Tabago & 0,3 & $-0,4$ \\
\hline \multicolumn{2}{|c|}{ Puertos con aumentos de actividad } & & 36,8 & \\
\hline \multirow{7}{*}{$\begin{array}{l}\frac{\infty}{\pi} \\
\frac{\pi}{0} \\
\frac{\pi}{0} \\
\frac{0}{0} \\
\frac{0}{2}\end{array}$} & Montevideo (100\%) & Uruguay & 1,4 & 0,1 \\
\hline & | Itajai (12\%) & Brasil & 2,3 & 1,9 \\
\hline & Guayaquil (91\%) & Ecuador & 3,6 & 2,6 \\
\hline & Zárate $(6 \%)$ & Argentina & 0,3 & 3,2 \\
\hline & Santos (38\%) & Brasil & 7,2 & 7,1 \\
\hline & Itapoa $(7 \%)$ & Brasil & 1,4 & 8,1 \\
\hline & Paranagua (8\%) & Brasil & 1,6 & 9,3 \\
\hline \multirow{4}{*}{ 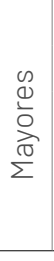 } & Rosario $(3 \%)$ & Argentina & 0,1 & 10,5 \\
\hline & Bahía de Cartagena (67\%) & Colombia & 5,4 & 11,7 \\
\hline & $\begin{array}{l}\text { Panamá, costa del Caribe, } \\
3 \text { terminales }(60 \%)\end{array}$ & Panamá & 8,1 & 14,0 \\
\hline & $\begin{array}{l}\text { Panamá, costa del Pacífico, } \\
2 \text { terminales }(39 \%)\end{array}$ & Panamá & 5,3 & 17,4 \\
\hline & Todos los puertos seleccionados & & 80,2 & $-0,8$ \\
\hline
\end{tabular}

Fuente: Comisión Económica para América Latina y el Caribe (CEPAL), sobre la base de información de operadores y autoridades portuarios.

- La creciente concentración ha redundado en procesos de integración vertical con los puertos. Por ejemplo, de acuerdo a datos de la CEPAL, a fines de marzo de 2020, las terminales integradas manejaban el $68 \%$ de los contenedores en Buenos Aires y el $49 \%$ en el Brasil. En el puerto de Callao (Perú), representan el $41 \%$ del total movilizado y en el Caribe el $35 \%$ del transbordo total.

- La súbita y prolongada caída del tráfico aéreo pone en riesgo a la industria y sus trabajadores y puede socavar la conectividad en la región. Las restricciones a los viajes se tradujeron en que la aviación haya sido uno de los sectores más afectados por la pandemia. Según la base de datos Data+ de la Organización de Aviación Civil Internacional (OACI), en mayo de 2020 los indicadores mundiales de tráfico aéreo de pasajeros (ingresos por pasajeros-kilómetros de pago (RPK)) y de carga aérea (toneladas-kilómetros de carga (FTK)) mostraban caídas interanuales del 98,9\% y el $62,1 \%$, respectivamente.

En la Argentina, el Brasil, Chile y Colombia, las caídas en mayo de 2020 fueron en promedio del $\mathbf{9 5 \%}$ (tráfico aéreo de pasajeros) y el $\mathbf{4 6 \%}$ (carga aérea). El hecho de que el volumen de 
carga transportada disminuyera menos en la región que a nivel mundial se explica por la baja participación de la logística aérea en el comercio internacional de la región. Esto permitió que, ante una demanda creciente y urgente de productos esenciales, algunas aerolíneas transformaran temporalmente sus aviones de pasajeros en cargueros aéreos, con lo que aumentaron los factores de capacidad y aminoraron la caída.

- La incertidumbre sobre la evolución de la pandemia y las medidas que deberá implementar la industria para retomar sus actividades, sumada a las precarias condiciones financieras de muchas empresas antes de la pandemia, ha llevado a la industria aérea a la peor crisis de su historia. Según la Asociación del Transporte Aéreo Internacional (IATA), la pérdida neta de las aerolíneas en 2020 podría alcanzar los 84.300 millones de dólares, lo que pondría en riesgo aproximadamente 32 millones de puestos de trabajo (IATA, 2020a). Desde el inicio de 2020 hasta fines de mayo, las aerolíneas perdieron casi la mitad de su valor de mercado a nivel mundial, mientras que en la región la caída llegó al 70\%.

- El fuerte impacto de la pandemia ha llevado a varias aerolíneas a solicitar apoyo financiero. Varios gobiernos del mundo comprometieron 123.000 millones de dólares en ayuda financiera al sector (IATA, 2020a). En los mercados emergentes, sin embargo, esas medidas han tenido un alcance relativamente limitado. Varias aerolíneas de la región (Aeroméxico, Avianca y LATAM) se vieron obligadas a iniciar procesos de reestructuración financiera en el marco del capítulo 11 de la Ley de quiebras de los Estados Unidos.

- Dado el papel crucial de la aviación en la conectividad territorial, el turismo y la creación de empleo, el futuro de las empresas del sector incidirá en el desempeño económico de la región. El impacto de la pandemia en los niveles de deuda de las aerolíneas, y sus posibles consecuencias sobre las rutas atendidas, las frecuencias y las tarifas de los servicios, pueden tener efectos negativos en la conectividad de los territorios extremos y la asequibilidad de sus servicios. Ello se debe a que muchas zonas insulares, alejadas o de difícil acceso dependen de los servicios aéreos para la movilidad de sus habitantes, la logística de sus cargas y la llegada de turistas.

- El cierre de actividades productivas, las medidas sanitarias más estrictas y los obstáculos administrativos han frenado el transporte terrestre. Pese a que el transporte por camión es crucial para satisfacer la demanda de artículos esenciales, se observa una menor actividad como consecuencia de la pandemia. A nivel mundial, se prevé que la disminución de la facturación anual de las empresas del sector en 2020 será de alrededor del 18\%; en América Latina alcanzaría al $20 \%$ (IRU, 2020). Estas proyecciones son concordantes con la caída del volumen transportado durante la primera mitad del año (véase el gráfico 12).

Gráfico 12 | América Latina (14 países): variación interanual del volumen de mercancías transportadas por camión, 2020 (En porcentajes)

A. Período marzo-mayo de 2020 en comparación con el mismo período de 2019

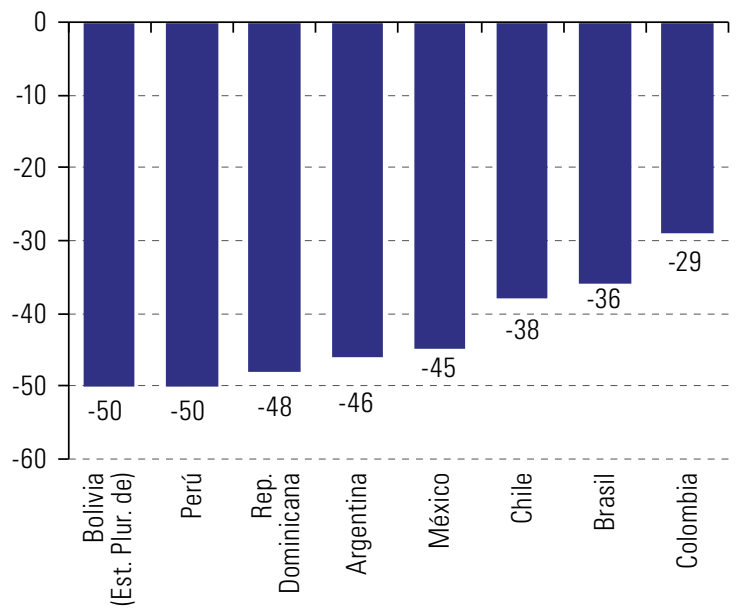

B. Período del 25 de febrero al 28 de junio de 2020 en comparación con el mismo período de $2019^{a}$

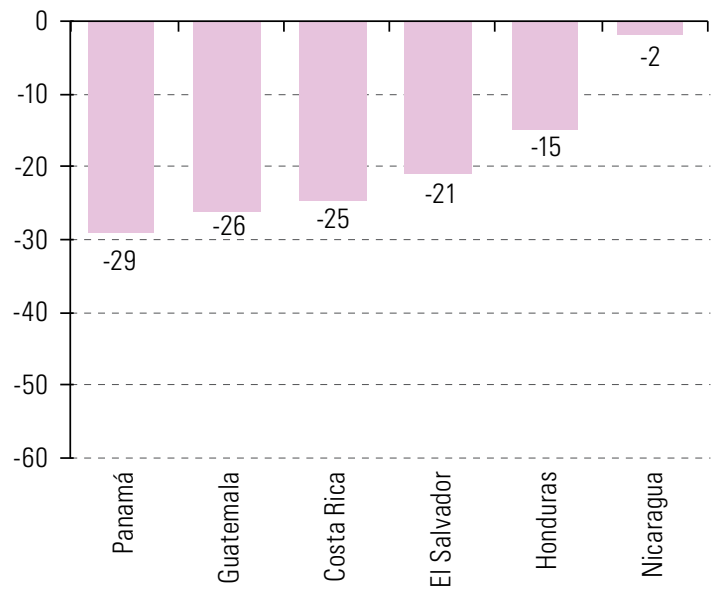

Fuente: Comisión Económica para América Latina y el Caribe (CEPAL), sobre la base de información de la Secretaría de Integración Económica Centroamericana (SIECA) y federaciones de camioneros.

a Número de declaraciones de tránsito terrestre por país de inicio en el sistema de Declaración Única Centroamericana (DUCA). 
- La disminución del volumen total transportado por camión se explica principalmente por la menor actividad económica y la paralización de sectores vinculados a la construcción, el turismo, el transporte de combustibles y las autopartes. Otro elemento que ha incidido en la menor actividad de transporte es el aumento de la duración de los ciclos de operación. Los tiempos de carga, descarga y tránsito se han incrementado debido a medidas como el establecimiento de barreras sanitarias en los principales corredores de transporte. Las demoras adicionales se han producido por problemas de facilitación de procesos, desconocimiento de protocolos de tránsito o la restricción de los plazos de permanencia de conductores extranjeros en cada país. Por ejemplo, en algunos casos las autoridades locales cerraron los accesos viales, prohibieron la apertura de recintos para prestar servicios sanitarios a los transportistas o incluso el descenso de estos en sus localidades para alimentarse o atender alguna emergencia con la carga.

- Al mismo tiempo que han disminuido los ingresos como consecuencia de las bajas de los fletes, han aumentado los retornos en vacío por la falta de carga en ambos extremos de la cadena de suministro. También se ha acentuado el distanciamiento temporal de los pagos, lo que reduce la liquidez de los transportistas, quienes, en su mayoría, operan como empresas unipersonales o microempresas. La informalidad característica del sector podría redundar en que no cumplieran con los requisitos necesarios para optar a ayudas financieras dirigidas a empresas.

- Se debe avanzar en la facilitación del comercio y de la logística internacional. Una logística eficiente y fluida es fundamental para asegurar el suministro oportuno de insumos y alimentos, así como para reducir distorsiones y sobreprecios que afectan desproporcionadamente a la población más vulnerable. Sin embargo, en algunos casos, para detener la propagación del virus se dictaron medidas restrictivas que afectaron el tránsito de mercancías e insumos entre países vecinos. Un ejemplo fue el cierre de fronteras por parte de Costa Rica, frente al riesgo derivado del aumento de los casos importados. Inmediatamente, los círculos empresariales y representantes del sector del transporte por camiones de los países vecinos alertaron sobre los impactos económicos de la medida. En respuesta, las autoridades centroamericanas aprobaron un protocolo de bioseguridad para el transporte de carga y comenzaron a implementar procedimientos coordinados para detener la propagación del coronavirus (véase SIECA, 2020).

- La logística urbana y el comercio electrónico han resultado vitales para la continuidad operativa de las actividades urbanas, al favorecer la distribución de alimentos y bienes necesarios para los largos períodos de confinamiento a los que ha estado sometida buena parte de la población. Un desafío en este sector es cómo proteger a los trabajadores de los riesgos de contagio y de la precariedad laboral en que gran parte de ellos se desempeñan, especialmente los migrantes. A mediano plazo, es urgente integrar la logística urbana a las políticas de transporte, reduciendo el número de viajes o haciéndolos más eficientes (por ejemplo, mediante la colaboración logística y el uso de medios con menores externalidades negativas, como la electromovilidad). Ello debería ir sumado a medidas que fortalezcan la coordinación de esas acciones con las de desarrollo territorial, uso del suelo y densificación de las ciudades.

\section{E. Las exportaciones y las importaciones de bienes se reducirían en una cuarta parte en 2020}

- Se estima que en el primer semestre del año los valores de las exportaciones y de las importaciones regionales de bienes habrían tenido caídas interanuales del $17 \%$ y el $18 \%$, respectivamente. El continuo deterioro de las perspectivas de crecimiento de varios de los principales mercados de la región y la incertidumbre sobre la evolución de la pandemia impiden prever una recuperación del comercio exterior regional en el segundo semestre.

- En este contexto, la Comisión Económica para América Latina y el Caribe (CEPAL) proyecta que en 2020 el valor de las exportaciones de bienes de la región se contraerá un $23 \%$, como resultado de caídas del precio (11\%) y del volumen (12\%) (véase el gráfico 13). En el caso del valor de las importaciones, la caída sería mayor (25\%) (véase el gráfico 14). La disminución del volumen importado alcanzaría el 18\%, de modo que sería algo mayor que la que registrada en 2009 durante la crisis financiera mundial y similar a las observadas en 1982, a comienzos de la crisis de la deuda externa, y en 1942, debido a la escasez resultante de la Segunda Guerra Mundial. 
Gráfico 13 | América Latina y el Caribe: variación de las exportaciones de bienes según valor, volumen y precio, $1930-2019$ y proyección para 2020

(En porcentajes)

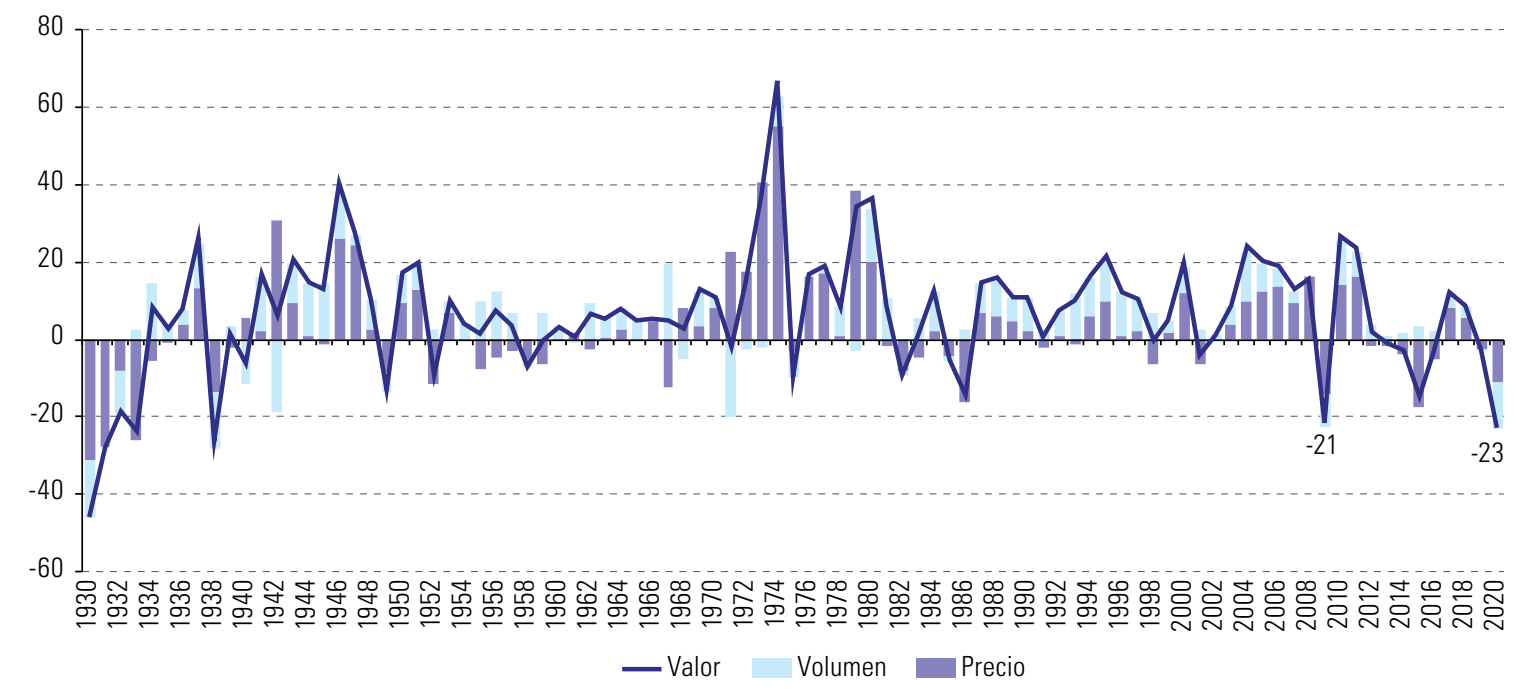

Fuente: Comisión Económica para América Latina y el Caribe (CEPAL), sobre la base de información oficial de la balanza de pagos de los países (1980-2019) y series de exportaciones a precios corrientes y de índices de precios de la CEPAL (1930-1979).

Nota: Las cifras de 2020 son proyecciones de la CEPAL.

Gráfico 14 | América Latina y el Caribe: variación de las importaciones de bienes según valor, volumen y precio, 1930-2019 y proyección para 2020

(En porcentajes)

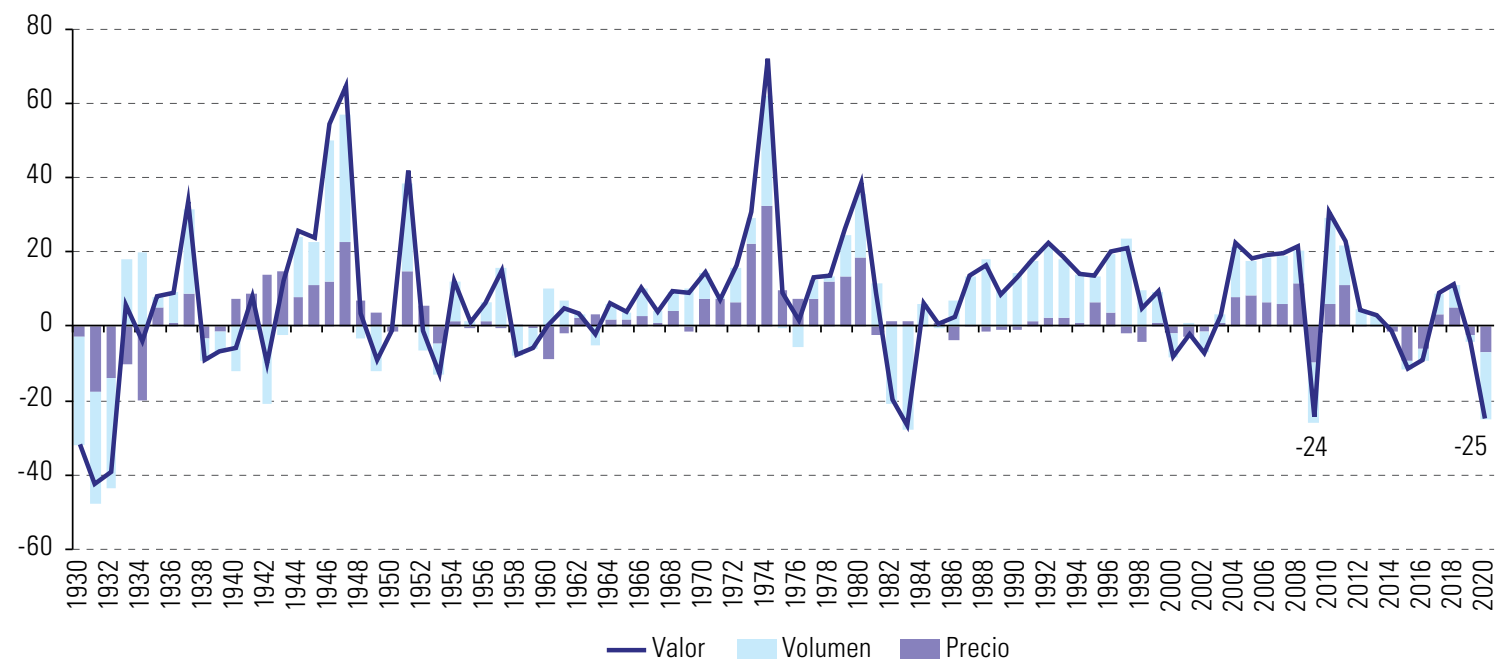

Fuente: Comisión Económica para América Latina y el Caribe (CEPAL), sobre la base de información oficial de la balanza de pagos de los países (1980-2019) y series de importaciones a precios corrientes y de índices de precios de la CEPAL (1930-1979).

Nota: Las cifras de 2020 son proyecciones de la CEPAL.

- Las mayores contracciones de las exportaciones en 2020 corresponderían a las destinadas a los Estados Unidos $(32 \%)$ y a la propia región $(28 \%)$, en tanto que los envíos a China caerían solo un $4 \%$ (véase el cuadro 13). Dada la composición de las exportaciones a esos mercados (principalmente manufacturas en los dos primeros casos y materias primas en el tercero), el efecto neto intensificaría la reprimarización de la canasta de exportaciones regional. Por su parte, las importaciones procedentes de todos los principales proveedores de la región tendrán caídas considerables. 
Cuadro 13 | América Latina y el Caribe: variación proyectada del valor del comercio de bienes, por socios, 2020 (En porcentajes)

\begin{tabular}{lcc}
\hline & Exportaciones & Importaciones \\
\hline Mundo & -23 & -25 \\
\hline Estados Unidos & -32 & -32 \\
\hline Unión Europea & -16 & -24 \\
\hline Asia & -7 & -23 \\
\hline China & -4 & -27 \\
\hline Resto de Asia & -11 & -19 \\
\hline América Latina y el Caribe & -28 & -29 \\
\hline
\end{tabular}

Fuente: Comisión Económica para América Latina y el Caribe (CEPAL), sobre la base de información de los bancos centrales, servicios de aduanas e institutos de estadística de los países.

De forma coherente con la evolución de las exportaciones por destino, se proyecta que las reducciones más fuertes se registren en los valores de los envíos de minería y petróleo y en los de manufacturas (véase el cuadro 14). En el primer caso, ello se debe en parte a una importante caída de los precios, mientras que en el segundo obedece fundamentalmente a los menores volúmenes exportados. Por su parte, los envíos agrícolas y agropecuarios serían los más resilientes, con un alza proyectada de su valor del $2 \%$. Las importaciones registrarán caídas considerables en todos los rubros, si bien la mayor contracción corresponderá a las compras de combustibles, debido a la disminución de los precios.

Cuadro 14 | América Latina y el Caribe: variación proyectada del valor del comercio de bienes, por sectores, 2020 (En porcentajes)

\begin{tabular}{lc}
\hline & Variación proyectada \\
\hline Exportaciones totales & -23 \\
\hline Productos agrícolas y agropecuarios & 2 \\
\hline Minería y petróleo & -33 \\
\hline Manufacturas & -25 \\
\hline Importaciones totales & -25 \\
\hline Bienes de capital & -19 \\
\hline Insumos intermedios & -21 \\
\hline Bienes de consumo & -22 \\
\hline Combustibles & -44 \\
\hline
\end{tabular}

Fuente: Comisión Económica para América Latina y el Caribe (CEPAL), sobre la base de información de los bancos centrales, servicios de aduanas e institutos de estadística de los países.

Dado que las importaciones caerían más que las exportaciones, la región llegaría a un saldo comercial superavitario de poco más de $\mathbf{4 5 . 0 0 0}$ millones de dólares en $\mathbf{2 0 2 0}$. Este se concentrará en los países del Mercado Común del Sur (MERCOSUR), Chile y México. Para la Comunidad Andina, cuyos miembros han sido fuertemente golpeados por la caída de los precios del petróleo y los minerales, se proyecta un déficit comercial de poco más de 4.500 millones de dólares. Por su parte, en los países centroamericanos y del Caribe se produciría una reducción importante del déficit respecto de 2019 (véase el gráfico 15).

- El pronunciado descenso de la demanda en la región redundaría en el desplome del comercio intrarregional en todos los rubros, casi todos los cuales presentarían disminuciones en porcentajes de dos dígitos (véase el cuadro 15). Esta situación repercutirá sobre todo en las exportaciones de manufacturas, cuyo principal mercado para casi todos los países es la propia región. 
Gráfico 15 | América Latina y el Caribe (agrupaciones y países seleccionados): saldo comercial de bienes, 2019 y proyección para 2020

(En miles de millones de dólares)

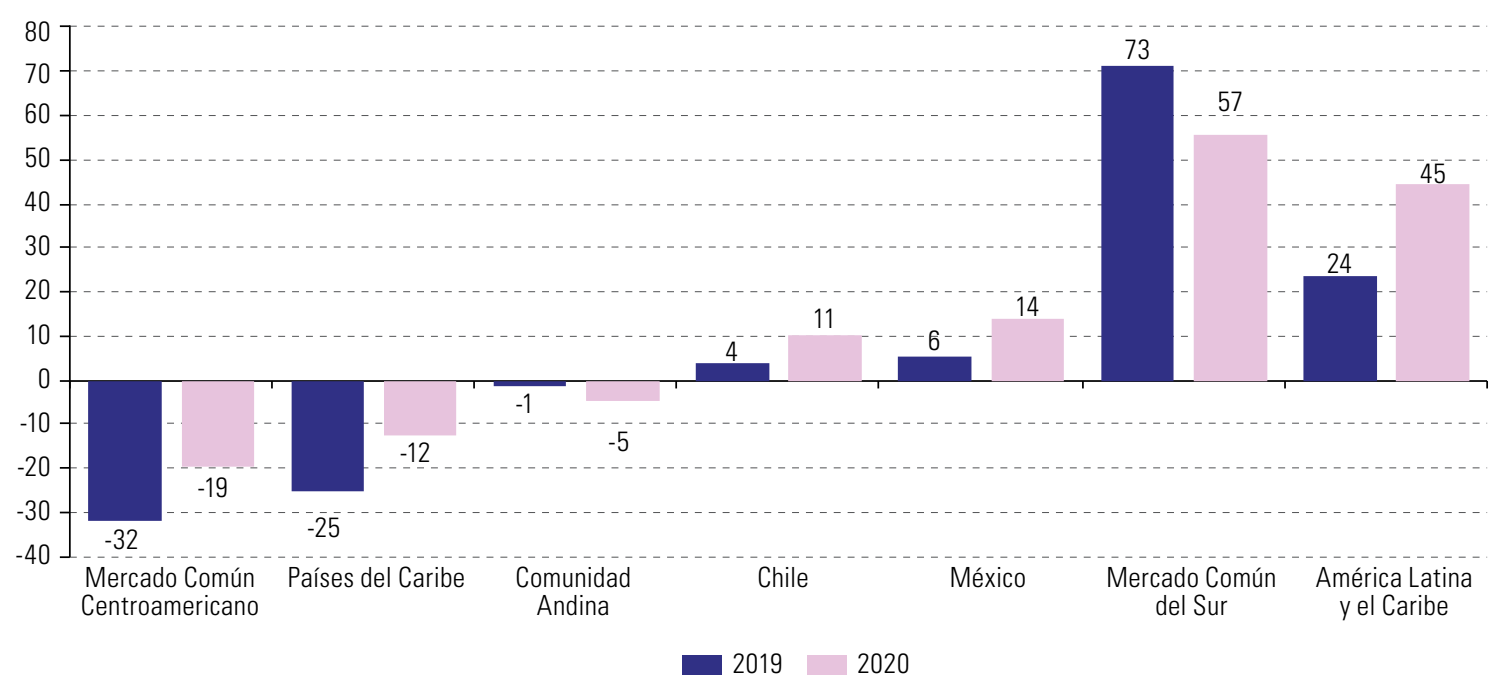

Fuente: Comisión Económica para América Latina y el Caribe (CEPAL), sobre la base de información oficial.

Cuadro 15 | América Latina y el Caribe: estructura del comercio intrarregional y coeficiente de comercio intrarregional medido por exportaciones, promedio 2018-2019, y variación proyectada para 2020a

(En millones de dólares y porcentajes)

\begin{tabular}{lcccc}
\hline Sector & $\begin{array}{c}\text { Exportaciones } \\
\text { intrarregionales }\end{array}$ & Participación & $\begin{array}{c}\text { Coeficiente } \\
\text { de comercio } \\
\text { intrarregional }\end{array}$ & $\begin{array}{c}\text { Variación } \\
\text { proyectada } \\
\text { para 2020 }\end{array}$ \\
\hline Agricultura, caza y pesca & 13521 & 8 & 10 & -2 \\
\hline Petróleo y minería & 25998 & 16 & 19 & -15 \\
\hline Alimentos, bebidas y tabaco & 19139 & 12 & 18 & -14 \\
\hline Madera, celulosa y papel & 5784 & 4 & 20 & -12 \\
\hline Textiles, confecciones y calzado & 6804 & 4 & 22 & -18 \\
\hline Química y petroquímica & 16505 & 10 & 41 & -24 \\
\hline Medicamentos & 3282 & 2 & 59 & -10 \\
\hline Caucho y plástico & 4643 & 3 & 35 & -24 \\
\hline Minerales no metálicos & 2408 & 1 & 93 & -23 \\
\hline Metales y productos derivados & 14383 & 9 & 8 & -27 \\
\hline Maquinaria y equipos & 16829 & 10 & 16 & -45 \\
\hline Automotor & 23418 & 14 & 13 & -33 \\
\hline Otras manufacturas & 11820 & 7 & 15 & -28 \\
\hline Total & 164535 & 100 & 93 & \\
\hline
\end{tabular}

Fuente: Comisión Económica para América Latina y el Caribe (CEPAL), sobre la base de información de UN Comtrade - Base de Datos Estadísticos sobre el Comercio Internacional.

a Se excluye la información de la industria maquiladora de Centroamérica.

- La evolución esperada del comercio exterior regional en 2020 apunta a una pérdida de capacidades productivas en el sector industrial, asociada a la fuerte contracción de las exportaciones manufactureras y de las importaciones de bienes de capital e insumos intermedios. Esto hará más difícil transitar hacia una especialización exportadora más intensiva en conocimientos a medida que la región emerja de la actual crisis. 


\section{F. Ante una economía mundial más incierta y regionalizada,}

es imperativo profundizar la integración regional

La pandemia probablemente reforzará dos tendencias interrelacionadas que ya se perfilaban previamente. La primera es una tendencia hacia un menor nivel de interdependencia productiva, comercial y tecnológica entre las principales economías mundiales, en particular entre los Estados Unidos y Europa, por una parte, y China, por la otra. La segunda es una tendencia hacia un comercio mundial con un menor nivel de apertura, más permeado por consideraciones geopolíticas y de seguridad nacional, con una mayor presencia de conflictos y con una institucionalidad multilateral debilitada. El resultado neto no sería una reversión de la globalización, sino una economía mundial más regionalizada, organizada en torno a tres grandes polos productivos: América del Norte, Europa y Asia Oriental y Sudoriental.

- En un contexto mundial de mayor regionalización de la producción, la integración regional debe desempeñar un papel clave en las estrategias de salida de la crisis en América Latina y el Caribe. Avanzar hacia el establecimiento de un mercado integrado de 650 millones de personas proporcionaría a los países de la región un importante seguro frente a perturbaciones de oferta o de demanda generadas fuera de ella. Asimismo, permitiría alcanzar la escala requerida para fortalecer industrias estratégicas como la farmacéutica y la de insumos médicos y promover redes de producción e investigación compartida entre países y subregiones. Esto reduciría la vulnerabilidad de la región ante interrupciones del suministro como las producidas durante la pandemia (CEPAL, 2020c). Por otra parte, en un escenario de debilitamiento del multilateralismo, una mayor concertación regional es indispensable para generar un diálogo en un mayor pie de igualdad con los principales actores de la economía mundial (véase el diagrama 1).

Diagrama 1 | Consecuencias inmediatas del COVID-19 en el comercio y la producción, y oportunidades para reforzar la integración regional en América Latina y el Caribe

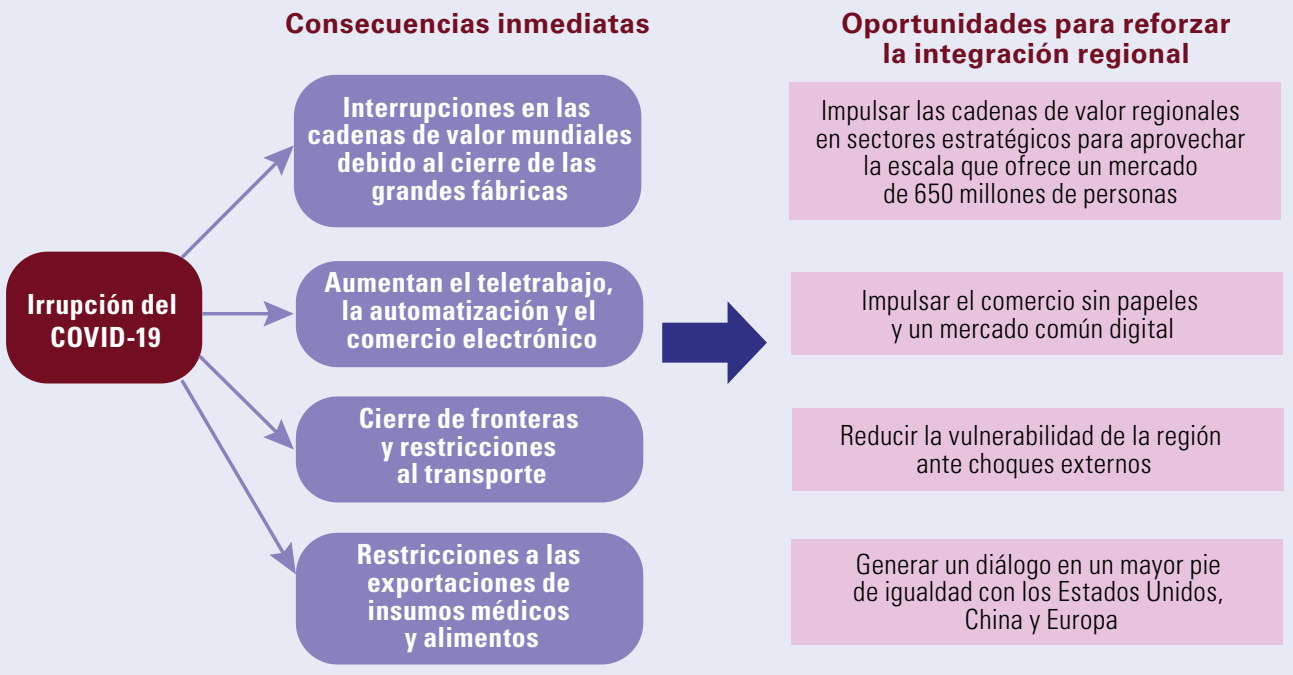

Fuente: Comisión Económica para América Latina y el Caribe (CEPAL).

Para avanzar en la integración regional, la infraestructura y la logística deben formar parte de los paquetes de medidas de recuperación económica. Además de su relevante participación directa en el PIB y el empleo, son fundamentales para la producción de todos los bienes y servicios, el suministro de alimentos y servicios esenciales, y la competitividad internacional del comercio.

- En el actual contexto de elevada incertidumbre, los países de la región deben emprender acciones que les permitan reducir sus costos logísticos internos y generar servicios de valor agregado para mantener su competitividad. Estas medidas deben implementarse de forma coordinada con otras medidas económicas y sociales, para favorecer una recuperación económica con beneficios sociales y ambientales. En el caso de la infraestructura y la logística, son cruciales cuatro elementos: inversión, interoperabilidad, integración regional e inteligencia logística. 
- La coyuntura actual es particularmente adversa para las iniciativas de profundización de la integración regional. A las diferencias de visión estratégica ya conocidas se suma ahora una crisis sanitaria, económica y social de enormes proporciones y el riesgo de una profunda crisis alimentaria. Sin embargo, no es posible ignorar las señales que apuntan a una marcada reconfiguración de la geografía económica global, y específicamente a una mayor relevancia de las regiones. Por ende, profundizar la integración regional debe ser un componente esencial de cualquier estrategia de salida de la crisis. Dado el rol crucial que el mercado regional tiene para las exportaciones de manufacturas y para las pequeñas y medianas empresas (pymes) exportadoras, emprender acciones coordinadas que propendan a la reactivación del comercio intrarregional permitirá atenuar la pérdida de tejido empresarial y la reprimarización de la estructura productiva y las exportaciones con que América Latina y el Caribe emergerá de la pandemia.

\section{Bibliografía}

Arias, J. (2020a), “Aumentan 11 \% a mayo 2020 las importaciones agroalimentarias de China, principalmente desde Estados Unidos y Argentina", Blog del Instituto Interamericano de Cooperación para la Agricultura (IICA), 29 de junio [en línea] https://blog.iica.int/index.php/blog/aumentan-12-las-importaciones-agricolaschina-principalmente-desde-alc.

(2020b), “Monitoreando el comercio agroalimentario durante el COVID-19", Blog del Instituto Interamericano de Cooperación para la Agricultura (IICA), 14 de julio [en línea] https://blog.iica.int/blog/estadisticascomercio-agricola-en-las-americas-covid-19.

Banco Mundial (2020), Global Economic Prospects: June 2020, Washington, D.C.

CEIE (Centro de Estudios para la Integración Económica) (2019), Informe Económico Regional 2018-2019, Ciudad de Guatemala, Secretaría de Integración Económica Centroamericana (SIECA).

CEPAL (Comisión Económica para América Latina y el Caribe) (2020a), "Enfrentar los efectos cada vez mayores del COVID-19 para una reactivación con igualdad: nuevas proyecciones," Informe Especial COVID-19, N 5, Santiago, julio.

(2020b), “Medidas de recuperación del sector turístico en América Latina y el Caribe: una oportunidad para promover la sostenibilidad y la resiliencia", Informes COVID-19, Santiago, julio.

(2020c), “Las restricciones a la exportación de productos médicos dificultan los esfuerzos por contener la enfermedad por coronavirus (COVID-19) en América Latina y el Caribe", Informes COVID-19, Santiago, mayo.

CIU (Cámara de Industrias del Uruguay) (2020), "Comercio exterior de bienes del Uruguay", Informe Mensual, año 11, N 124, 12 de mayo [en línea] http://www.ciu.com.uy/innovaportal/file/90088/1/abril2020-n124.pdf.

IATA (Asociación de Transporte Aéreo Internacional) (2020a), "Economic performance of the airline industry", junio [en línea] https://www.iata.org/en/iata-repository/publications/economic-reports/airlineindustry-economic-performance-june-2020-report/.

(2020b), "Airlines Financial Monitor", junio [en línea] https://www.iata.org/en/iata-repository/publications/ economic-reports/airlines-financial-monitor--may-20202/.

INDEC (Instituto Nacional de Estadística y Censos) (2020), “Intercambio comercial argentino: cifras estimadas de mayo de 2020", Informes Técnicos, vol. 4, No 114 [en línea] https://www.indec.gob.ar/ uploads/informesdeprensa/ica_06_204EC2AC1D23.pdf.

INEGI (Instituto Nacional de Estadística y Geografía) (2020), “Información oportuna sobre la balanza comercial de mercancías de México durante mayo de 2020", Comunicado de Prensa, N 286/20, 26 de junio [en línea] https://www.inegi.org.mx/contenidos/saladeprensa/boletines/2020/balcom_o/balcom_ o2020_06.pdf.

IRU (International Road Transport Union) (2020), COVID-19 Impacts on the Road Transport Industry. Executive Summary, junio.

Ministerio de Economía del Brasil (2020), “Balança comercial brasileira: acumulado do ano" [en línea] http:// www.mdic.gov.br/index.php/comercio-exterior/estatisticas-de-comercio-exterior/balanca-comercialbrasileira-acumulado-do-ano. 
OMC (Organización Mundial del Comercio) (2020a), “El comercio se contrae de forma acusada en la primera mitad de 2020", Comunicado de Prensa, N 858, 22 de junio [en línea] https://www.wto.org/ spanish/news_s/pres20_s/pr858_s.htm.

_(2020b), "E-commerce, trade and the COVID-19 pandemic: information note", 4 de mayo [en línea] https://www.wto.org/english/tratop_e/covid19_e/ecommerce_report_e.pdf.

OMT (Organización Mundial del Turismo) (2020), "New data shows impact of COVID-19 on tourism as UNWTO calls for responsible restart of the sector", 22 de junio [en línea] https://www.unwto.org/news/ new-data-shows-impact-of-covid-19-on-tourism.

SIECA (Secretaría de Integración Económica Centroamericana) (2020), “Lineamientos de bioseguridad sector transporte CA" [en línea] https://www.sieca.int/index.php/lineamientos-bioseguridad-sectortransporte-terrestre-ca/.

Este Informe Especial es el sexto de una serie que elabora la Comisión Económica para América Latina y el Caribe (CEPAL) sobre la evolución y los efectos de la pandemia de COVID-19 en América Latina y el Caribe. Sus análisis económicos y sociales se actualizarán a medida que surja información relevante. La Secretaria Ejecutiva de la CEPAL, Alicia Bárcena, dirigió la elaboración de este Informe, con el apoyo técnico de la Oficina del Secretario Ejecutivo Adjunto, Mario Cimoli, y de la División de Comercio Internacional e Integración de la CEPAL.

Copyright (C) Naciones Unidas, 2020 